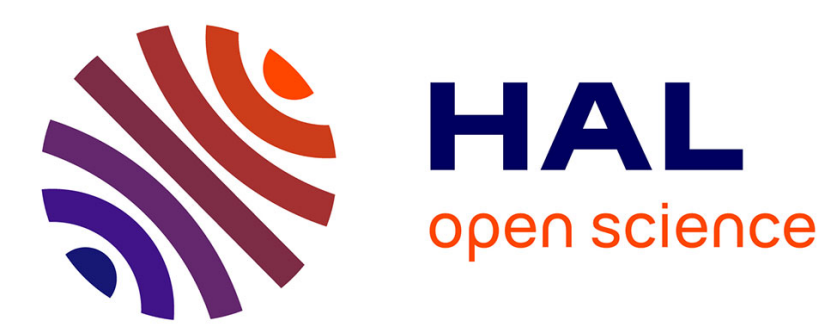

\title{
Sur une question d'Erdös et Schinzel
}

Gérald Tenenbaum

\section{To cite this version:}

Gérald Tenenbaum. Sur une question d'Erdös et Schinzel. 1990, pp.405-443. hal-00021869

\section{HAL Id: hal-00021869 \\ https://hal.science/hal-00021869}

Submitted on 27 Mar 2006

HAL is a multi-disciplinary open access archive for the deposit and dissemination of scientific research documents, whether they are published or not. The documents may come from teaching and research institutions in France or abroad, or from public or private research centers.
L'archive ouverte pluridisciplinaire HAL, est destinée au dépôt et à la diffusion de documents scientifiques de niveau recherche, publiés ou non, émanant des établissements d'enseignement et de recherche français ou étrangers, des laboratoires publics ou privés. 
Paru dans A Tribute to Paul Erdös,

A. Baker, B. Bollobás, A. Hajnal (eds.)

Cambridge University Press (1990), 405-443.

(C) Cambridge University Press, 1990.

\title{
Sur une question d'Erdős et Schinzel*
}

\author{
Gérald Tenenbaum
}

A Paul Erdös, qui ouvre la voie.

\section{Introduction}

Désignons par $P^{+}(m)$ le plus grand facteur premier d'un entier générique $m$, avec la convention $P^{+}(1)=1$. Un théorème de Tchébychev, publié en 1895 , après sa mort, par Markov, énonce que

$$
x^{-1} P^{+}\left(\prod_{n \leqslant x}\left(n^{2}+1\right)\right) \rightarrow \infty \quad(x \rightarrow \infty) .
$$

Il est naturel de conjecturer beaucoup plus : si la suite polynomiale

$$
\left\{n^{2}+1: n=1,2, \ldots\right\}
$$

contient effectivement son quota heuristique de nombres premiers, on doit avoir pour $x$ assez grand

$$
P^{+}\left(\prod_{n \leqslant x}\left(n^{2}+1\right)\right) \gg x^{2}
$$

Ce problème a suscité, par le passé, la curiosité de nombreux mathématiciens et fait encore régulièrement, de nos jours, l'objet d'intéressantes publications. En 1952, Erdős [5] a montré que, pour tout polynôme irréductible $F(X)$ à coefficients entiers, on a

$$
P^{+}\left(\prod_{n \leqslant x} F(n)\right)>x \exp \left\{c \log _{2} x \log _{3} x\right\} \quad\left(x>x_{0}(F)\right) .
$$

\footnotetext{
* Nous incluons ici certaines corrections par rapport à la version publiée.
} 
(Ici et dans toute la suite de cet article, nous notons $\log _{k}$ la $k$-ième itérée de la fonction logarithme.)

Dans le cas des polynômes quadratiques, un progrès important a été accompli par Hooley [9] qui, en 1967, a obtenu dans (1.1) la minoration $x^{1+\mu}$ avec $\mu=\frac{1}{10}$ - cf. également le chapitre 2 de [10]. Le meilleur résultat actuellement connu dans cette direction est dû à Deshouillers \& Iwaniec [2], avec une constante $\mu$ excédant légèrement $\frac{1}{5}$.

Toutefois, en l'absence de majoration suffisamment fine pour certaines sommes de Kloostermann incomplètes, ces dernières estimations n'ont pas d'équivalent en degré plus grand que 2. Récemment, Erdős \& Schinzel se sont réattaqués à cette question et ont montré [6] que l'on peut remplacer la borne inférieure de $(1 \cdot 2)$ par

$$
x \exp \exp \left\{c\left(\log _{2} x\right)^{1 / 3}\right\}
$$

où $c$ est une constante absolue positive. Cette évaluation a pu être obtenue grâce à une amélioration du Lemma 1 d'Erdös [5], que l'on peut formuler ainsi : Désignons, pour $x \geqslant z \geqslant y \geqslant 2$, par $H_{F}(x, y, z)$ le nombre des entiers $n$ n'excédant pas $x$ pour lesquels $F(n)$ possède au moins un diviseur d tel que $y<d \leqslant z$. Alors

$$
H_{F}\left(x, \frac{1}{2} x, x\right)>\frac{x}{\log x} \exp \left\{c^{\prime}\left(\log _{2} x\right)^{1 / 3}\right\}
$$

De plus, tout renforcement de $(1 \cdot 3)$ conduit par la même méthode à une amélioration correspondante dans le problème de Tchébychev.

Dans cette situation, Erdős \& Schinzel ont formulé le problème, d'intérêt propre, de l'évaluation asymptotique de $H_{F}(x, y, z)$ sans restriction de primalité sur le polynôme $F$ et pour toutes valeurs relatives des variables $x, y$ et $z$. Ils posent en particulier la question de déterminer pour quels polynômes $F$ la densité

$$
D_{F}(y):=\lim _{x \rightarrow \infty} x^{-1} H_{F}(x, y, 2 y)
$$

tend vers 0 lorsque $y \rightarrow \infty$.

Nous nous proposons ici d'aborder l'étude asymptotique de $H_{F}(x, y, z)$. Par souci de simplicité, nous n'envisageons que le cas $z \leqslant 2 y$. Nous définissons alors implicitement la quantité $\beta=\beta(y, z)$ par la formule

$$
z=y\left\{1+(\log y)^{-\beta}\right\} .
$$

Notre approximation pour $H_{F}(x, y, z)$ est de la forme

$$
x(\log y)^{-\delta(\beta, F)}
$$


où l'exposant $\delta(\beta, F)$ est optimal, en un sens que nous préciserons ultérieurement. Quelques notations sont nécessaires pour définir explicitement cette quantité.

Considérons un polynôme $F(X)$ à coefficients entiers, prenant des valeurs positives sur les entiers positifs, et dont une décomposition en produit de facteurs irréductibles dans $\mathbb{Z}[X]$ (essentiellement unique) est

$$
F(X)=\prod_{j=1}^{r} F_{j}(X)^{\alpha_{j}}
$$

Par analogie avec les fonctions arithmétiques classiques, nous posons

$$
\widehat{\omega}(F):=r, \quad \widehat{\Omega}(F):=\sum_{j=1}^{r} \alpha_{j}, \quad \widehat{\tau}(F):=\prod_{j=1}^{r}\left(\alpha_{j}+1\right) .
$$

Un rôle particulier est joué par la fonction de variable réelle

$$
\gamma(v)=\gamma_{F}(v):=\sum_{j=1}^{r}\left\{\left(\alpha_{j}+1\right)^{v}-1\right\}
$$

(cf. en particulier le lemme 4.1). Nous désignons par $u=u(\beta, F)$ l'unique solution de l'équation

$$
\gamma_{F}^{\prime}(u)=\sum_{j=1}^{r}\left(\alpha_{j}+1\right)^{u} \log \left(\alpha_{j}+1\right)=\max \left\{\beta+1, \gamma_{F}^{\prime}(0)\right\}
$$

(noter que $\gamma_{F}^{\prime}(0)=\log \widehat{\tau}(F)$ ) et nous définissons $\delta(\beta, F)$ par la formule

$$
\delta(\beta, F):= \begin{cases}u \gamma_{F}^{\prime}(u)-\gamma_{F}(u) & \left(0 \leqslant \beta \leqslant \gamma_{F}^{\prime}(1)-1\right), \\ \beta+1-\gamma_{F}(1) & \left(\beta>\gamma_{F}^{\prime}(1)-1\right) .\end{cases}
$$

Il est peut-être plus agréable de transformer cette expression en introduisant la fonction

$$
Q(t):=t \log t-t+1 \quad(t>0)
$$

qui est positive ou nulle et possède un unique zéro (double) au point $t=1$. On peut alors écrire

$$
\delta(\beta, F)= \begin{cases}\sum_{j=1}^{r} Q\left(\left(\alpha_{j}+1\right)^{u}\right) & (0 \leqslant u \leqslant 1), \\ \beta+1-\widehat{\Omega}(F) & (u>1) .\end{cases}
$$

Il est à noter que $\delta(\beta, F)$ est continue en $\beta=\gamma_{F}^{\prime}(1)-1$. On a $\delta(\beta, F) \geqslant 0$ pour tout $\beta \geqslant 0$, avec égalité si et seulement si $\beta+1 \leqslant \log \widehat{\tau}(F)$. 
Théorème. Soient $\beta_{0}$ et $B$ deux nombres réels positifs. Il existe des constantes positives $y_{0}, c_{j}(0 \leqslant j \leqslant 5)$, ne dépendant que de $\beta_{0}, B$ et $F$, telles que les propositions suivantes soient vérifiées pour $y_{0} \leqslant y \leqslant x^{c_{0}}$ et $0 \leqslant \beta(y, z) \leqslant B$.

(a) Si $\beta+1 \leqslant \log \widehat{\tau}(F)-c_{1} / \sqrt{\log _{2} y}$, alors

$$
c_{2} x \leqslant H_{F}(x, y, z) \leqslant\left(1-c_{2}\right) x .
$$

(b) Si $\beta+1>\log \widehat{\tau}(F)-c_{1} / \sqrt{\log _{2} y}$, alors

$$
c_{3} x(\log y)^{-\delta(\beta, F)} \mathrm{e}^{-c_{4} \sqrt{\log _{2} y \cdot \log _{3} y}} \leqslant H_{F}(x, y, z) \leqslant c_{5} x(\log y)^{-\delta(\beta, F)} .
$$

De plus, le terme $\log _{3} y$ peut être omis dans le membre de gauche lorsque $\beta \geqslant \beta_{0}$.

Complément. Quitte à altérer les valeurs de $y_{0}$ et $c_{j}(1 \leqslant j \leqslant 5)$, la minoration de (1.13) et l'encadrement (1.14) sont valables pour tout $c_{0}<1$.

Remarque. On a $\widehat{\tau}(F) \geqslant 3$ dès que $F$ est réductible. Quitte à choisir $\beta_{0}<\log 3-1$, l'hypothèse du point (b) du théorème implique alors $\beta \geqslant \beta_{0}$.

Lorsque $F$ est irréductible, on a en particulier

$$
H_{F}(x, y, 2 y)=x(\log y)^{-\delta+o(1)}
$$

pour $x$ et $y$ tendant vers $+\infty$ dans tout domaine $y \leqslant x^{c_{0}}\left(c_{0}<1\right)$, avec

$$
\delta:=\delta(0, F)=1-\left(\frac{1+\log \log 2}{\log 2}\right) \approx 0,086071 .
$$

Cela conduirait à une amélioration considérable de (1·3) si l'on pouvait choisir $c_{0}=1$. Malheureusement, la méthode du présent travail repose de manière essentielle sur l'estimation par le crible de quantités du type

$$
\operatorname{card}\{n \leqslant x: d|F(n), p| F(n) \Rightarrow p \mid d \text { ou } p>t\}
$$

où $d>y$ et $t$ est un paramètre «grand». Cela interdit a priori de considérer des valeurs de $y$ de l'ordre de $x$. Le complément au théorème représente donc pratiquement la limite naturelle de notre méthode. En l'absence d'une profonde altération du raisonnement suivi dans [5], un tel résultat semble inutilisable dans le problème de Tchébychev — voir cependant la note p. 444 .

Dans [5], Erdős avait suggéré qu'il était possible d'obtenir une borne inférieure du type

$$
P^{+}\left(\prod_{n \leqslant x} F(n)\right)>x \exp \left\{(\log x)^{c}\right\}
$$

Cette inégalité découlerait en fait, pour tout $c<1-\delta$, de la minoration contenue dans (1.15) si la valeur $y=x$ était admissible.

Dans le cas des «petites》 valeurs de $y$, nos résultats permettent de répondre complètement à la question d'Erdős et Schinzel concernant la densité (1.4). 
Corollaire. $D_{F}(y)$ tend vers 0 à l'infini si, et seulement si, $F(X)$ est irréductible dans $\mathbb{Z}[X]$. Dans ce cas, on a de plus

$$
D_{F}(y)=(\log y)^{-\delta+o(1)} \quad(y \rightarrow \infty)
$$

où $\delta$ est défini par (1·16).

Lorsque $F$ est réductible, on a $c_{2}(F) \leqslant D_{F}(y) \leqslant 1-c_{2}(F)$, et Erdős conjecture que $D_{F}(y)$ tend vers une limite $\ell(F)$, nécessairement dans $] 0,1[$. Nos méthodes ne semblent pas permettre l'obtention d'un tel résultat.

Le point (b) du théorème généralise essentiellement l'étude faite dans l'article [18] et dans le chapitre 2 de [8], qui correspond au cas $F(X)=X$. Nous avions alors également considéré les «grandes》 valeurs de $z$, i.e. $z>2 y$. Dans cette circonstance, les nombres premiers $\leqslant z / y$ jouent un rôle particulier engendrant des difficultés techniques supplémentaires que nous avons préféré éviter ici. Leur présence ne modifie cependant pas la méthode dans son principe et il serait tout-à-fait possible, le cas échéant, d'étendre dans cette direction le champ de validité de nos estimations. De même, on pourrait s'affranchir de la condition $\beta(y, z) \leqslant B$ en adaptant convenablement la méthode élémentaire présentée au début du paragraphe 2.5 de [8]. Il est d'ailleurs vraisemblable que l'on obtienne ainsi un équivalent asymptotique de $H_{F}(x, y, z)$ si $\beta(y, z)$ ne tend pas trop vite vers l'infini.

Le changement de comportement asymptotique de $H_{F}(x, y, z)$ au passage par la valeur critique

$$
\beta=\gamma_{F}^{\prime}(1)-1=\sum_{j=1}^{r}\left(\alpha_{j}+1\right) \log \left(\alpha_{j}+1\right)-1
$$

est susceptible d'une interprétation probabiliste. Lorsque $z$ est suffisamment proche de $y$, les différentes conditions $F(n) \equiv 0(\bmod d)(y<d \leqslant z)$, sont essentiellement indépendantes et l'on a

$$
H_{F}(x, y, z) \approx \sum_{n \leqslant x} \tau(F(n) ; y, z),
$$

où $\tau(m ; y, z)$ désigne le nombre de diviseurs de $m$ dans l'intervalle $] y, z]$. En revanche, lorsque $z / y$ dépasse un certain seuil, l'effet de la dépendance est perceptible et $H_{F}(x, y, z)$ devient notablement moindre que le membre de droite de $(1 \cdot 18)$. Le lecteur trouvera dans la section 2.2 de [8] une discussion plus approfondie sur ce point, contenant notamment, dans le cas $F(X)=X$, une 
justification heuristique de la valeur $\beta=2 \log 2-1$ du seuil de dépendance. Le raisonnement s'étend sans difficulté au cas général.

La première partie du théorème appelle encore une remarque. Considérons, pour fixer les idées, le cas $F(X)=X^{2}, \beta=0, y$ fixé. Nous obtenons que, pour une densité positive (minorée indépendamment de $y$ ) d'entiers $n$, le nombre $n^{2}$ possède un diviseur dans $\left.] y, 2 y\right]$. Cette propriété équivaut clairement à l'existence de deux diviseurs $d$ et $d^{\prime}$ de $n$ tels que $y<d d^{\prime} \leqslant 2 y$. Le problème apparaît donc comme une version duale de la conjecture d'Erdős, résolue dans [13], selon laquelle il est «presque toujours» (i.e. : dans une suite de densité 1) possible de trouver $d$ et $d^{\prime}$ tels que $1<d^{\prime} / d \leqslant 2$. Dans les deux cas, le résultat est suggéré par l'hypothèse heuristique d'équiprobabilité des quantités $\log \left(d d^{\prime}\right)$ ou $\log \left(d^{\prime} / d\right)$ - puisque le nombre des diviseurs de $n^{2}$, comme celui des rapports distincts $d^{\prime} / d$, est normalement $(\log n)^{\log 3+o(1)}$. Or, la majoration de (1.13) indique les limites de cette analogie : alors que la conjecture d'Erdős est effectivement satisfaite «presque partout», la propriété relative aux diviseurs de $n^{2}$ n'a pas lieu sur un ensemble de densité 1 ou même proche de 1 . Il faut voir une explication de ce phénomène dans le principe, souvent observé, qu'un ensemble-différence est mieux réparti qu'un ensemble-somme.

L'auteur a le plaisir de remercier ici Paul Erdős, Andrzej Schinzel, Léo Murata et Jean-Marie De Koninck pour leur aide durant la préparation de cet article.

\section{Notations et conventions - rappels sur les congruences algébriques}

Les lettres $p$ et $q$ sont réservées pour désigner exclusivement des nombres premiers. On note $P^{-}(n)$ (resp. $P^{+}(n)$ ) le plus petit (resp. le plus grand) facteur premier de l'entier $n>1$. Par convention, $P^{-}(1)=+\infty, P^{+}(1)=1$.

Nous désignons par $\tau(n)$ (resp. $\omega(n)$ ) le nombre des diviseurs (resp. des facteurs premiers) de l'entier $n$, et nous définissons, pour chaque valeur du paramètre réel $\vartheta$, les fonctions arithmétiques multiplicatives

$$
\begin{gathered}
\tau(n, \vartheta):=\sum_{d \mid n} d^{i \vartheta}, \\
\tau_{\vartheta}(n):=\prod_{\substack{p^{\nu} \| n \\
p \leqslant \exp (1 /|\vartheta|)}}(\nu+1) .
\end{gathered}
$$


Pour tous $y$ et $z$ tels que $1 \leqslant y \leqslant z$, nous posons également

$$
\tau(n ; y, z)=\sum_{\substack{d \mid n \\ y<d \leqslant z}} 1 .
$$

Étant donné un entier $n \geqslant 1$, nous notons $d \mid n^{\infty}$ la propriété $: p|d \Rightarrow p| n$.

Nous employons indifféremment les symboles $O$ de Landau et $\ll$ de Vinogradov. L'écriture $A \asymp B$ signifie : $A \ll B$ et $B \ll A$.

Dans tout l'article, nous supposons fixé un polynôme $F(X)$ à coefficients entiers, de degré $g \geqslant 1$, prenant des valeurs positives sur les entiers positifs, et dont la décomposition canonique dans $\mathbb{Z}[X]$ est fournie par (1.6). Nous posons également

$$
F^{*}(X):=\prod_{j=1}^{r} F_{j}(X)
$$

Pour chaque polynôme $G(X)$ de $\mathbb{Z}[X]$, nous désignons par $\varrho(n ; G)$ le nombre des solutions modulo $n$ de la congruence

$$
G(x) \equiv 0 \quad(\bmod n) .
$$

Il découle du théorème chinois que $\varrho(n ; G)$ est une fonction multiplicative de $n$. Pour la concision, nous notons

$$
\varrho(n):=\varrho(n ; F), \quad \varrho_{j}(n):=\varrho\left(n ; F_{j}\right)(1 \leqslant j \leqslant r), \quad \varrho^{*}(n):=\varrho\left(n ; F^{*}\right) .
$$

Soit $D^{*}$ le discriminant de $F^{*}(X)$. On sait que

$$
\varrho^{*}\left(p^{\nu}\right)=\varrho^{*}(p) \quad\left(p \nmid D^{*}, \nu \geqslant 1\right)
$$

— cf. par exemple Nagell [15], theorem 52. Comme l'égalité $\varrho^{*}(p)=p$ n'est possible que si $p \mid F(1)$, on peut écrire

$$
\varrho^{*}\left(p^{\nu}\right) \leqslant \min \{g, p-1\} \quad\left(p \nmid D^{*} F(1), \nu \geqslant 1\right) .
$$

Pour la commodité du lecteur, nous rappelons maintenant quelques faits simples qui sont pour la plupart établis dans [3].

Lorsque $1 \leqslant i<j \leqslant r$, les polynômes $F_{i}(X)^{\alpha_{i}}$ et $F_{j}(X)^{\alpha_{j}}$ sont premiers entre eux dans $\mathbb{Z}[X]$. Nous désignons par $m_{i j} \in \mathbb{Z}$ leur pgcd, c'est-à-dire le plus petit entier positif de la forme

$$
m_{i j}=U(X) F_{i}(X)^{\alpha_{i}}+V(X) F_{j}(X)^{\alpha_{j}},
$$


avec $U(X), V(X) \in \mathbb{Z}[X]$. Nous posons alors

$$
M:=\prod_{1 \leqslant i<j \leqslant r} m_{i j}
$$

avec la convention $M=1$ si $r=1$. Il est clair que les congruences $F_{i}(x) \equiv 0(\bmod p)$ et $F_{j}(x) \equiv 0(\bmod p)$ sont incompatibles lorsque $p \nmid m_{i j}$. D'où

$$
\varrho\left(p^{\nu}\right)=\sum_{j=1}^{r} \varrho\left(p^{\nu}, F_{j}^{\alpha_{j}}\right) \quad(p \nmid M) .
$$

Maintenant, on vérifie facilement que l'on a pour tous $p, \nu \geqslant 1,1 \leqslant j \leqslant r$,

$$
\varrho\left(p^{\nu}, F_{j}^{\alpha_{j}}\right)=p^{\nu-\left\lceil\nu / \alpha_{j}\right\rceil} \varrho_{j}\left(p^{\left\lceil\nu / \alpha_{j}\right\rceil}\right)
$$

où, ici et dans tout l'article, nous notons $\lceil x\rceil$ le plus petit entier au moins égal à $x \in \mathbb{R}$.

Nous posons une fois pour toutes

$$
D:=D^{*} F(1) M \text {. }
$$

Ainsi l'ensemble des facteurs premiers de $D$ contient tous les nombres premiers pour lesquels la fonction $\varrho\left(p^{\nu}\right)$ est susceptible de posséder un comportement exceptionnel. Il découle en particulier de (2.5), (2.8) et (2.9) que l'on a

$$
\varrho\left(p^{\nu}\right)=\sum_{j=1}^{r} \varrho_{j}(p) p^{\nu-\left\lceil\nu / \alpha_{j}\right\rceil} \quad(p \nmid D, \nu \geqslant 1) .
$$

Nous associons également à $F^{*}(X)$ une «fonction d'Euler»

$$
\varphi^{*}(n):=n \prod_{p \mid n}\left(1-\varrho^{*}(p) / p\right)
$$

Par (2.6), on a

$$
\varphi^{*}(n) \geqslant 1 \quad(n \geqslant 1,(n, D)=1) .
$$

Il nous sera utile dans la suite de disposer d'une majoration uniforme pour $\varrho\left(p^{\nu}\right)$. Nous la déduirons, comme dans [3], du résultat de Nagell \& Ore cf. [15], theorem 54

$$
\varrho^{*}\left(p^{\nu}\right) \leqslant g \cdot\left(D^{*}\right)^{2} \quad(p \geqslant 2, \nu \geqslant 1) .
$$


Cela implique

$$
\varrho\left(p^{\nu}\right) \ll \sum_{j=1}^{r} p^{\nu-\left\lceil\nu / \alpha_{j}\right\rceil}
$$

lorsque $p \nmid M$ (grâce à (2.8) et (2.9)). Nous allons voir que cette estimation persiste en fait même lorsque $p \mid M$. Supposons en effet que $p^{\mu} \| M$. Nous pouvons certainement nous restreindre au cas $\nu>\mu$ r. Alors la relation $p^{\nu} \mid F(n)$ implique $p^{\mu+1} \mid F_{j}(n)^{\alpha_{j}}$ pour au moins un $j$. La plus grande puissance de $p$ divisant le produit de tous les autres $F_{i}(n)^{\alpha_{i}}$ est nécessairement $\leqslant \mu$, donc $j$ est unique. On en déduit que $p^{\nu-\mu} \mid F_{j}(n)^{\alpha_{j}}$, et il suit

$$
\begin{aligned}
\varrho\left(p^{\nu}\right) & \leqslant p^{\mu} \sum_{j=1}^{r} \varrho\left(p^{\nu-\mu} ; F_{j}^{\alpha_{j}}\right) \\
& =\sum_{j=1}^{r} p^{\nu-\left\lceil(\nu-\mu) / \alpha_{j}\right\rceil} \varrho_{j}\left(p^{\left\lceil(\nu-\mu) / \alpha_{j}\right\rceil}\right) .
\end{aligned}
$$

$\mathrm{Au}$ vu de (2.14), cela implique bien (2.15).

\section{Arithmétique des suites polynomiales - lemmes généraux}

Nous nous proposons dans cette section d'établir les principaux résultats auxiliaires, nécessaires à la démonstration de notre théorème, et qui concernent le comportement asymptotique de fonctions multiplicatives restreintes à des suites polynomiales. Bien entendu les fonctions $\varrho_{j}(p)$ s'avèrent fondamentales, et les trois premiers lemmes leur sont dévolus.

Lemme 3.1. Soit $G(X)$ un polynôme irréductible de $\mathbb{Z}[X]$. On a pour $x$ infini

$$
\sum_{p \leqslant x} \varrho(p ; G)=\operatorname{li}(x)+O\left(x \mathrm{e}^{-c \sqrt{\log x}}\right)
$$

où $c=c(G)$ est une constante positive. 
Démonstration. Nous nous contentons de brèves indications pour cette estimation classique. Soit $K=\mathbb{Q}(\xi)$ le corps de nombres engendré par une racine de l'équation $G(\xi)=0$. Désignons par $\mathcal{O}$ l'anneau des entiers de $K$, par $N$ la norme de $K \operatorname{sur} \mathbb{Q}$, et par $\wp$ un idéal premier générique de $\mathcal{O}$. Le théorème des idéaux premiers sous une forme forte (cf. Landau [11], Satz 191) s'écrit

$$
\sum_{N \wp \leqslant x} 1=\operatorname{li}(x)+O\left(x \mathrm{e}^{-c \sqrt{\log x}}\right) .
$$

Or on a, pour chaque $\wp, N \wp=p^{f}$ où $p$ est un nombre premier rationnel. Comme l'a remarqué Erdős dans [4], la contribution à (3.2) des $\wp$ tels que $p$ divise le discriminant $D_{G}$ de $G$ est $O(1)$, et celle des $\wp$ tels que $f>1$ est $O(\sqrt{x})$ puisque le nombre des $\wp$ associés à un $p$ fixé ne dépasse pas le degré de $G$. Lorsque $f=1, p \nmid D_{G}$, on a

$$
\operatorname{card}\{\wp: N \wp=p\}=\varrho(p ; G)
$$

puisque ce nombre est exactement celui des facteurs linéaires distincts dans la réduction de $G \bmod p-$ cf. par exemple Lang [12], chap. I, §8. Cela suffit pleinement à établir (3.1).

Lemme 3.2. On a pour $x$ infini

$$
\sum_{p \leqslant x} \varrho^{*}(p) / p=r \log _{2} x+O(1) .
$$

Démonstration. Cela découle immédiatement de (2.11) et (3.1), par sommation d'Abel.

Lemme 3.3. Soit $f$ une fonction périodique de période $2 \pi$, à variation bornée sur l'intervalle $[0,2 \pi[$, et de valeur moyenne

$$
\bar{f}:=\frac{1}{2 \pi} \int_{0}^{2 \pi} f(t) d t .
$$

Pour tout polynôme irréductible $G$ de $\mathbb{Z}[X]$, il existe une constante positive $c=c(G)$ telle que l'on ait pour $\vartheta \in \mathbb{R}, \vartheta \neq 0,2 \leqslant y \leqslant x$,

$$
\begin{aligned}
& \sum_{y<p \leqslant x} \varrho(p ; G) \frac{f(\vartheta \log p)}{p} \\
& \quad=\bar{f} \log \left(\frac{\log x}{\log y}\right)+O\left(\frac{V(f)}{|\vartheta| \log y}+\frac{M(f)+(1+|\vartheta|) V(f)}{e^{c \sqrt{\log y}}}\right)
\end{aligned}
$$


où l'on a posé $V(f):=\int_{0}^{2 \pi}|d f(t)|, M(f):=\sup _{0 \leqslant t \leqslant 2 \pi}|f(t)|$.

La démonstration est identique à celle du Lemma 30.1 de [8], à ceci près que $\pi(x)$ doit être remplacé par la fonction sommatoire de $\varrho(p ; G)$. Le lemme 3.1 remplaçant le théorème des nombres premiers, les calculs sont inchangés.

Le résultat suivant est une application simple de la théorie du crible.

Lemme 3.4. Désignons par $d_{0}, d_{1}, \ldots, d_{r}$ des nombres entiers deux à deux premiers entre eux et tels que

$$
d_{0} \mid D^{\infty}, \quad\left(d_{j}, D\right)=1 \quad(1 \leqslant j \leqslant r) .
$$

Soient $K, K^{\prime}$, des nombres réels satisfaisant à $0<K<1 \leqslant K^{\prime}$. Il existe une constante positive $c_{6}=c_{6}(K, F)<1$ telle que, sous la condition

$$
2 \leqslant y \leqslant x^{c_{6}}, \quad d_{0} d_{1} \ldots d_{r} \leqslant x^{1-K}, \quad P^{+}\left(d_{0} d_{1} \ldots d_{r}\right) \leqslant y^{K^{\prime}}
$$

on ait uniformément

$$
\sum_{\substack{\left.n \leqslant x \\ d_{j}\left|F_{j}(n) \\ d_{0}\right| F(n), j \leqslant r\right) \\ p|F(n) \Rightarrow p| D \prod_{j=1}^{r} d_{j} \text { ou } p>y}} 1 \asymp \frac{x}{(\log y)^{r}} \frac{\varrho\left(d_{0}\right)}{d_{0}} \prod_{j=1}^{r} \frac{\varrho_{j}\left(d_{j}\right)}{\varphi^{*}\left(d_{j}\right)} .
$$

De plus, la relation $(3 \cdot 5)$ persiste, en remplaçant le signe $\asymp$ par $\ll$, lorsque $P^{+}\left(d_{0} d_{1} \ldots d_{r}\right)>y^{K^{\prime}}$.

Démonstration. On peut supposer

$$
\varrho\left(d_{0}\right) \prod_{j=1}^{r} \varrho_{j}\left(d_{j}\right) \geqslant 1,
$$

car le résultat est trivial dans le cas contraire. On applique alors le crible de Selberg à l'ensemble d'entiers

$$
\mathcal{A}:=\left\{F^{*}(n): n \leqslant x, d_{0}\left|F(n), d_{j}\right| F_{j}(n)(1 \leqslant j \leqslant r)\right\}
$$

pour l'ensemble de nombres premiers

$$
\mathcal{P}:=\left\{p: p \leqslant y, p \nmid D \prod_{j=1}^{r} d_{j}\right\} .
$$


Par le théorème chinois, on a

$$
|\mathcal{A}|=X+O(R)
$$

avec

$$
\left\{\begin{array}{l}
X:=x \frac{\varrho\left(d_{0}\right)}{d_{0}} \prod_{j=1}^{r} \frac{\varrho_{j}\left(d_{j}\right)}{d_{j}} \geqslant x^{K} \varrho\left(d_{0}\right), \\
R:=\varrho\left(d_{0}\right) \prod_{j=1}^{r} \varrho_{j}\left(d_{j}\right) \ll x^{K / 3} \varrho\left(d_{0}\right),
\end{array}\right.
$$

où la seconde estimation découle de (2.6). Lorsque $d \mid \prod_{p \in \mathcal{P}} p$, on a similairement (notant, comme c'est l'usage, $\mathcal{A}_{d}$ l'ensemble des multiples de $d$ qui appartiennent à $\mathcal{A}$ )

$$
\left|\mathcal{A}_{d}\right|=X \frac{\varrho^{*}(d)}{d}+O\left(R \varrho^{*}(d)\right)
$$

avec

$$
\varrho^{*}(d) \leqslant g^{\omega(d)} \quad\left(d \mid \prod_{p \in \mathcal{P}} p\right) .
$$

Le lemme fondamental du crible de Selberg, tel qu'il est énoncé, par exemple, par Halberstam et Richert dans [7] (theorem 7.1) implique alors que, pour tout choix du paramètre $v \geqslant 1$, le membre de gauche de (3.5) vaut

$$
X \prod_{p \in \mathcal{P}}\left(1-\frac{\varrho^{*}(p)}{p}\right)\left\{1+O\left(\mathrm{e}^{-v \log v}\right)\right\}+O\left(R \sum_{d \leqslant y^{2 v}}(3 g)^{\omega(d)}\right)
$$

(La condition traditionnelle « $\Omega_{2}(\kappa) »$ est ici trivialement impliquée par (3.7), avec $\kappa=g$.) Choisissons $v$ assez grand pour que le terme entre accolades soit dans l'intervalle $\left[\frac{1}{2}, \frac{3}{2}\right]$, puis $c_{6}$ assez petite pour que le dernier terme d'erreur soit $\ll x^{K / 2} \varrho\left(d_{0}\right)$. Cela est possible, compte tenu de (3.6), puisque l'on a classiquement

$$
\sum_{d \leqslant z} b^{\omega(d)} \ll_{b} z(\log 2 z)^{b-1} \quad(z \geqslant 1, b>0)
$$

Observons ensuite que, puisque $\varrho^{*}(p) \leqslant \min (p-1, g)$ lorsque $p \in \mathcal{P}$, le terme principal de (3.8) est alors 


$$
\geqslant \frac{1}{2} X \prod_{\substack{p \leqslant y \\ p \nmid D}}\left(1-\frac{\varrho^{*}(p)}{p}\right) \gg \frac{x^{K} \varrho\left(d_{0}\right)}{(\log x)^{r}} .
$$

Cela implique que l'expression (3.8) est

$$
\asymp X \prod_{p \in \mathcal{P}}\left(1-\frac{\varrho^{*}(p)}{p}\right) .
$$

Le résultat annoncé découle donc de (3.3).

Nous allons maintenant utiliser le résultat précédent pour majorer des sommes de fonctions multiplicatives d'arguments polynomiaux. Nous avons en fait besoin d'une estimation uniforme lorsque la sommation est restreinte aux entiers $n$ tels que $F(n) \equiv 0 \quad(\bmod d)$. Il serait théoriquement possible d'étendre stricto sensu les travaux d'Ennola [3] ou de Wolke [19], tous deux reposant sur la méthode développée par Erdős [4] pour évaluer $\sum_{n \leqslant x} \tau(G(n))$ lorsque $G(X)$ est irréductible. Un tel résultat, qui pourrait être l'analogue du théorème de Shiu [17], serait fort utile et mériterait certainement un article pour lui-même. En tout état de cause, la généralisation n'est pas de pure routine et induit de notables difficultés techniques. Nous nous sommes, pour ces raisons, cantonné ici à une version plus simple, mais cependant suffisante pour l'application que nous envisageons. Les calculs sont grandement facilités par l'hypothèse de forte multiplicativité imposée aux fonctions arithmétiques considérées.

Lemme 3.5. Soit $A$ une constante positive arbitraire et $h_{1}, h_{2}, \ldots, h_{r}$ des fonctions arithmétiques fortement multiplicatives satisfaisant pour tout nombre premier $p$ à

$$
0 \leqslant h_{j}(p) \leqslant A \quad(1 \leqslant j \leqslant r) .
$$

Soit $K$ un nombre réel, $0<K<1$. On a, uniformément pour $x \geqslant 1$ et $1 \leqslant d \leqslant x^{1-K}$,

$$
\sum_{\substack{n \leqslant x \\ d \mid F(n)}} \prod_{j=1}^{r} h_{j}\left(F_{j}(n)\right) \ll x \frac{g(d)}{d} \exp \left\{\sum_{j=1}^{r} \sum_{p \leqslant x} \frac{\varrho_{j}(p)\left(h_{j}(p)-1\right)}{p}\right\}
$$

où $g$ est la fonction multiplicative définie par

$$
g\left(p^{\nu}\right)= \begin{cases}\varrho\left(p^{\nu}\right) & \text { si } p \mid D, \\ \frac{1}{1-\varrho^{*}(p) / p} \sum_{j=1}^{r} \varrho_{j}(p) h_{j}(p) p^{\nu-\left\lceil\nu / \alpha_{j}\right\rceil} & \text { si } p \nmid D .\end{cases}
$$


Démonstration. Pour chaque entier $n$ tel que $d \mid F(n)$, on définit les ensembles de nombres premiers

$$
\mathcal{P}_{j}(n):=\left\{p: p \mid F_{j}(n), p \nmid D\right\} \quad(1 \leqslant j \leqslant r) .
$$

Ainsi que nous l'avons remarqué au paragraphe 2 , les $\mathcal{P}_{j}(n)$ sont deux à deux disjoints. Notant $\mathcal{P}_{0}$ l'ensemble des facteurs premiers de $D$, on voit que $\left\{\mathcal{P}_{0}, \mathcal{P}_{1}(n), \ldots, \mathcal{P}_{r}(n)\right\}$ induit une partition de l'ensemble des facteurs premiers de $d$. Nous lui associons la décomposition

$$
d=d_{0} \prod_{j=1}^{r} d_{j}(n) \quad\left(d_{0}=\prod_{\substack{p^{\nu} \| d \\ p \mid D}} p^{\nu}, d_{j}(n)=\prod_{\substack{p^{\nu} \| d \\ p \in \mathcal{P}_{j}(n)}} p^{\nu}(1 \leqslant j \leqslant r)\right) .
$$

Considérons maintenant un $r$-uple $\left\{d_{1}, d_{2}, \ldots, d_{r}\right\}$ d'entiers deux à deux premiers entre eux tels que $d_{1} d_{2} \ldots d_{r}=d / d_{0}$ et un entier $n$ tel que $d_{j}(n)=d_{j}$ $(1 \leqslant j \leqslant r)$. L'hypothèse $d \mid F(n)$ implique

$$
m_{j} \mid F_{j}(n) \quad(1 \leqslant j \leqslant r)
$$

avec

$$
m_{j}:=\prod_{p^{\nu} \| d_{j}} p^{\left\lceil\nu / \alpha_{j}\right\rceil} .
$$

Le membre de gauche de (3.9), disons $S$, satisfait donc à l'inégalité

$$
S \leqslant \sum_{\substack{d_{1} \ldots d_{r}=d / d_{0} \\\left(d_{i}, D d_{j}\right)=1}} S\left(d_{1}, \ldots, d_{r}\right)
$$

avec

$$
S\left(d_{1}, \ldots, d_{r}\right):=\sum_{\substack{n \leqslant x \\ m_{j}\left|F_{j}(n)(1 \leqslant j \leqslant r) \\ d_{0}\right| F(n)}} \prod_{j=1}^{r} h_{j}\left(F_{j}(n)\right) .
$$

Ici et dans toute la suite de cette démonstration, $m_{j}$ est défini en fonction de $d_{j} \operatorname{par}(3.11)$.

Pour chaque $n$ apportant une contribution positive à $S\left(d_{1}, \ldots, d_{r}\right)$, on peut décomposer $F_{j}(n)(1 \leqslant j \leqslant r)$ de manière unique sous la forme

$$
F_{j}(n)=D_{n j} M_{n j} A_{n j} B_{n j}
$$

avec les conditions

$$
\begin{aligned}
D_{n j} \mid D^{\infty}, \quad & m_{j}\left|M_{n j}\right| m_{j}^{\infty}, \quad\left(A_{n j} B_{n j}, D m_{j}\right)=1, \\
& P^{+}\left(A_{n j}\right) \leqslant \xi<P^{-}\left(B_{n j}\right),
\end{aligned}
$$


où $\xi=\xi(n)$ est un paramètre que nous préciserons plus loin. Notons $d_{n j}, a_{n j}$ et $b_{n j}$ les noyaux sans facteur carré respectifs de $D_{n j}, A_{n j}$ et $B_{n j}$. On a alors

$$
h_{j}\left(F_{j}(n)\right)=h_{j}\left(d_{n j}\right) h_{j}\left(m_{j}\right) h_{j}\left(a_{n j}\right) h_{j}\left(b_{n j}\right),
$$

et il sera utile dans la suite de garder à l'esprit la majoration

$$
h_{j}\left(d_{n j}\right) \leqslant A^{\omega(D)} \ll 1 \quad(1 \leqslant j \leqslant r) .
$$

Posons maintenant

$$
\widetilde{a}_{n}:=\prod_{j=1}^{r} a_{n j}, \quad \widetilde{b}_{n}:=\prod_{j=1}^{r} b_{n j}, \quad q_{n}:=P^{-}\left(\widetilde{b}_{n}\right)
$$

et

$$
X=x^{c_{7}}
$$

avec $c_{7}=\min \left\{K, c_{6}\left(\frac{1}{2} K, F\right)\right\}$. Nous choisissons, pour chaque $n, \xi=\xi(n)$ aussi grand que possible sous la contrainte

$$
\widetilde{a}_{n} \leqslant \sqrt{X} .
$$

On a donc nécessairement

$$
\widetilde{a}_{n} q_{n}>\sqrt{X} .
$$

Nous désignons respectivement par $S_{1}\left(d_{1}, \ldots, d_{r}\right)$ et $S_{2}\left(d_{1}, \ldots, d_{r}\right)$ les contributions à $S\left(d_{1}, \ldots, d_{r}\right)$ des entiers $n \leqslant x$ tels que $\widetilde{a}_{n} \leqslant X^{1 / 3}$ et $\widetilde{a}_{n}>X^{1 / 3}$.

Pour majorer $S_{1}\left(d_{1}, \ldots, d_{r}\right)$, nous utilisons l'inégalité (3·15) sous la forme $q_{n}>\sqrt{X} / \widetilde{a}_{n} \geqslant X^{1 / 6}$. En remarquant que $h_{j}\left(b_{n j}\right) \ll A^{6 g / c_{7}} \ll 1$, il suit

$$
S_{1}\left(d_{1}, \ldots, d_{r}\right) \ll \prod_{j=1}^{r} h_{j}\left(m_{j}\right) \quad \sum_{\substack{a_{1} \ldots a_{r} \leqslant X \\\left(a_{i}, D m_{i} a_{j}\right)=1}}^{\prime} \prod_{\substack{1 \leqslant i \neq j \leqslant r) \\ j=1}}^{r} h_{j}\left(a_{j}\right) \sum_{n \leqslant x}^{(\dagger)} 1
$$

avec les conditions de sommation

$$
\text { (†) }\left\{\begin{array}{l}
a_{j} m_{j} \mid F_{j}(n)(1 \leqslant j \leqslant r) \\
d_{0} \mid F(n) \\
p|F(n) \Rightarrow p| D \prod_{j=1}^{r} a_{j} m_{j} \text { ou } p>X^{1 / 6}
\end{array}\right.
$$

Ici et dans la suite de cette section, l'apostrophe indique que la sommation ainsi désignée est restreinte à des entiers sans facteur carré. Puisque

$$
d_{0} \prod_{j=1}^{r} a_{j} m_{j} \leqslant x^{1-K / 2},
$$


on peut appliquer le lemme 3.4 pour estimer la somme intérieure. On a ainsi

$$
\sum_{n \leqslant x}^{(\dagger)} 1 \ll \frac{x}{(\log x)^{r}} \frac{\varrho\left(d_{0}\right)}{d_{0}} \prod_{j=1}^{r} \frac{\varrho_{j}\left(a_{j} m_{j}\right)}{\varphi^{*}\left(a_{j} m_{j}\right)} .
$$

En remarquant que

$$
\begin{aligned}
\sum_{\substack{a_{1} \ldots a_{r} \leqslant X \\
\left(a_{1} \ldots a_{r}, D\right)=1}}^{\prime} \prod_{j=1}^{r} \frac{h_{j}\left(a_{j}\right) \varrho_{j}\left(a_{j}\right)}{\varphi^{*}\left(a_{j}\right)} & \leqslant \prod_{j=1}^{r} \sum_{\substack{a \leqslant X \\
(a, D)=1}}^{\prime} \frac{\varrho_{j}(a) h_{j}(a)}{\varphi^{*}(a)} \\
& \leqslant \exp \left(\sum_{j=1}^{r} \sum_{p \leqslant x}\left\{\frac{\underline{\varrho}_{j}(p) h_{j}(p)}{p}+O\left(\frac{1}{p^{2}}\right)\right\}\right),
\end{aligned}
$$

on obtient finalement

$$
S_{1}\left(d_{1}, \ldots, d_{r}\right) \ll x \frac{\varrho\left(d_{0}\right)}{d_{0}} \prod_{j=1}^{r} \frac{\varrho_{j}\left(m_{j}\right) h_{j}\left(m_{j}\right)}{\varphi^{*}\left(m_{j}\right)} \exp \left\{\sum_{j=1}^{r} \sum_{p \leqslant x} \frac{\varrho_{j}(p)\left(h_{j}(p)-1\right)}{p}\right\}
$$

où nous avons fait appel au lemme 3.2 pour transformer le facteur $(\log x)^{-r}$ dans $(3 \cdot 16)$.

Désignons le facteur initial d'indice $j$ dans $(3 \cdot 17)$ par $G_{j}\left(d_{j}\right)$. On a

$$
G_{j}\left(d_{j}\right)=\prod_{\substack{p \mid d_{j} \\ p^{\nu} \| d}} G_{j}\left(p^{\nu}\right)
$$

d'où

$$
\sum_{\substack{d_{1} \ldots d_{r}=d / d_{0} \\\left(d_{i}, d_{j}\right)=1}} \prod_{(1 \leqslant i<j \leqslant r)}^{r} G_{j}\left(d_{j}\right)=\prod_{p^{\nu} \| d / d_{0}} \sum_{j=1}^{r} G_{j}\left(p^{\nu}\right)=\frac{d_{0}}{d} g\left(\frac{d}{d_{0}}\right) .
$$

Compte tenu de (3.17), cela montre que la contribution à $S$ des $S_{1}\left(d_{1}, \ldots, d_{r}\right)$ est compatible avec l'estimation annoncée $(3 \cdot 9)$.

Pour évaluer la contribution des $S_{2}\left(d_{1}, \ldots, d_{r}\right)$, nous utilisons toujours l'identité $(3 \cdot 14)$, mais nous majorons $h_{j}\left(b_{n j}\right)$ en fonction de $q:=P^{+}\left(\widetilde{a}_{n}\right)$. Puisque $b_{n j} \ll x^{g}$, on a pour $x$ assez grand

$$
\omega\left(b_{n j}\right)=\sum_{\substack{p \mid b_{n j} \\ p>q}} 1 \leqslant(g+1) \frac{\log x}{\log q} .
$$

D'où

$$
\prod_{j=1}^{r} h_{j}\left(b_{n j}\right) \leqslant x^{C / \log q}
$$


avec

$$
C:=r(g+1) \max \{0, \log A\}
$$

Il vient ainsi

$$
S_{2}\left(d_{1}, \ldots, d_{r}\right) \ll \prod_{j=1}^{r} h_{j}\left(m_{j}\right) \sum_{\substack{q \leqslant \sqrt{X} \\ q \nmid D}} x^{C / \log q} \sum_{a_{1}, \ldots, a_{r}}^{\prime(\ddagger)} \prod_{j=1}^{r} h_{j}\left(a_{j}\right) \sum_{n \leqslant x}^{(*)} 1
$$

avec les conditions de sommation

$$
(\ddagger)\left\{\begin{array}{l}
X^{\frac{1}{3}}<a_{1} \ldots a_{r} \leqslant X^{1 / 2} \\
\left(a_{i}, D m_{i} a_{j}\right)=1 \\
P^{+}\left(a_{1} \ldots a_{r}\right)=q
\end{array} \quad(1 \leqslant i, j \leqslant r, i \neq j)\right.
$$

et

$$
(*)\left\{\begin{array}{l}
a_{j} m_{j} \mid F_{j}(n) \quad(1 \leqslant j \leqslant r) \\
d_{0} \mid F(n) \\
p|F(n) \Rightarrow p| D \prod_{j=1}^{r} a_{j} m_{j} \text { ou } p>q .
\end{array}\right.
$$

Par le lemme 3.4, on a

$$
\sum_{n \leqslant x}^{(*)} 1 \ll \frac{x}{(\log q)^{r}} \frac{\varrho\left(d_{0}\right)}{d_{0}} \prod_{j=1}^{r} \frac{\varrho_{j}\left(a_{j} m_{j}\right)}{\varphi^{*}\left(a_{j} m_{j}\right)}
$$

En reportant dans $(3 \cdot 20)$ et en faisant appel à $(3 \cdot 18)$, il suit

$$
S_{2}:=\sum_{\substack{d_{1} \ldots d_{r}=d / d_{0} \\\left(d_{i}, d_{j}\right)=1}} S_{2}\left(d_{1}, \ldots, d_{r}\right) \ll x \frac{g(d)}{d} \sum_{q \leqslant \sqrt{X}} x^{C / \log q} \frac{T_{q}}{(\log q)^{r}}
$$

avec

$$
T_{q}:=\sum_{a_{1}, \ldots, a_{r}}^{\prime(\ddagger)} \prod_{j=1}^{r} \frac{\varrho_{j}\left(a_{j}\right) h_{j}\left(a_{j}\right)}{\varphi^{*}\left(a_{j}\right)} .
$$

En regroupant la somme $r$-uple suivant les valeurs de $a=a_{1} \ldots a_{r}$, on peut écrire

$$
T_{q} \leqslant \sum_{\substack{X^{1 / 3}<a \leqslant X^{1 / 2} \\ P^{+}(a)=q \\(a, D)=1}}^{\prime} \frac{\vartheta(a)}{a},
$$

où $\vartheta$ est la fonction multiplicative définie sur les entiers sans facteur carré par 


$$
\vartheta(p)=\frac{1}{1-\varrho^{*}(p) / p} \sum_{j=1}^{r} \varrho_{j}(p) h_{j}(p) .
$$

On a en particulier pour tout $\alpha>0$

$$
\begin{aligned}
T_{q} & \leqslant \frac{\vartheta(q)}{q} \sum_{\substack{X^{1 / 3} q^{-1}<b \leqslant X^{1 / 2} q^{-1} \\
P^{+}(b) \leqslant q,(b, D)=1}}^{\prime} \frac{\vartheta(b)}{b}\left(\frac{b q}{X^{1 / 3}}\right)^{\alpha} \\
& \ll q^{\alpha-1} X^{-\alpha / 3} \prod_{\substack{p \leqslant q \\
p \nmid D}}\left(1+\vartheta(p) p^{\alpha-1}\right) \\
& \ll q^{\alpha-1} X^{-\alpha / 3} \exp \left\{\sum_{j=1}^{r} \sum_{p \leqslant q} \frac{\varrho_{j}(p) h_{j}(p)}{p} p^{\alpha}\left\{1+O\left(\frac{1}{p}\right)\right\}\right\}
\end{aligned}
$$

Choisissons $\alpha=6 C / c_{7} \log q$. On a pour chaque $j, 1 \leqslant j \leqslant r$,

$$
\sum_{p \leqslant q} \frac{\varrho_{j}(p) h_{j}(p)}{p}\left\{p^{\alpha}\left(1+O\left(p^{-1}\right)\right)-1\right\} \ll \sum_{p \leqslant q} \frac{\varrho_{j}(p)\{\alpha \log p+1 / p\}}{p} \ll 1
$$

où nous avons fait appel au lemme 3.1, après sommation d'Abel. Nous pouvons donc écrire

$$
T_{q} \ll x^{-2 C / \log q} q^{-1} \exp \left\{\sum_{j=1}^{r} \sum_{p \leqslant x} \frac{\varrho_{j}(p) h_{j}(p)}{p}\right\} .
$$

En reportant dans $(3 \cdot 21)$, on constate donc que la somme en $q$ est

$$
\ll T \exp \left\{\sum_{j=1}^{r} \sum_{p \leqslant x} \frac{\varrho_{j}(p)\left(h_{j}(p)-1\right)}{p}\right\}
$$

avec

$$
T:=\sum_{q \leqslant x} x^{-C / \log q}\left(\frac{\log x}{\log q}\right)^{r} q^{-1} \ll 1 .
$$

Cela achève la démonstration.

Le résultat suivant est une conséquence facile du lemme 3.5. Il montre, dans le cas $d=1$, que le membre de gauche de (3.9) est dominé des entiers $n$ tel que le produit des «petits》 facteurs premiers de $F(n)$ est lui-même «petit».

Lemme 3.6. Dans les hypothèses du lemme 3.5, on a uniformément pour $w \geqslant v \geqslant 2$ et $x \geqslant 2$, 


$$
\begin{aligned}
\sum\left\{\prod_{j=1}^{r} h_{j}\left(F_{j}(n)\right)\right. & \left.: n \leqslant x, \prod_{j=1}^{r} \prod_{\substack{p \mid F_{j}(n) \\
p \leqslant v}} p>w\right\} \\
& \ll x \exp \left\{-\frac{\log w}{\log v}+\sum_{j=1}^{r} \sum_{p \leqslant x} \frac{\varrho_{j}(p)\left(h_{j}(p)-1\right)}{p}\right\} .
\end{aligned}
$$

Démonstration. On applique le lemme 3.5 aux fonctions fortement multiplicatives $g_{j}(1 \leqslant j \leqslant r)$ définies par

$$
g_{j}(p)= \begin{cases}h_{j}(p) p^{\alpha} & \text { si } p \leqslant v \\ h_{j}(p) & \text { si } p>v\end{cases}
$$

avec $\alpha:=1 / \log v$. On a ainsi $g_{j}(p) \leqslant e A$ pour tout $p$ et tout $j$. La somme à majorer n'excède pas

$$
w^{-\alpha} \sum_{n \leqslant x} \prod_{j=1}^{r} g_{j}\left(F_{j}(n)\right)
$$

et le résultat découle de (3.9) - avec $g_{j}$ à la place de $h_{j}$ — puisque l'on a pour chaque $j$

$$
\sum_{p \leqslant v} \frac{\varrho_{j}(p)\left(h_{j}(p)-1\right)\left(p^{\alpha}-1\right)}{p} \ll \sum_{p \leqslant v} \varrho_{j}(p) \frac{\alpha \log p}{p} \ll 1 .
$$

Nous aurons également besoin de la variante suivante du lemme 3.6, dans laquelle $h_{j} \equiv 1$ mais où les petits facteurs premiers sont comptés avec multiplicité. Nous n'avons pas recherché la forme optimale du résultat — le point essentiel étant ici la complète uniformité. Il est certainement possible d'améliorer l'estimation dans un domaine convenable en $v, w$ - cf. par exemple [20].

Lemme 3.7. On a uniformément pour $w \geqslant v \geqslant 2, x \geqslant 2$,

$$
\operatorname{card}\left\{n \leqslant x: \prod_{\substack{p^{\nu} \| F(n) \\ p \leqslant v}} p^{\nu}>w\right\} \ll x \exp \left\{-c_{8} \frac{\log w}{\log v}\right\}
$$

où $c_{8}=c_{8}(F)$ est une constante positive.

Démonstration. Posons $\alpha=\max _{1 \leqslant j \leqslant r} \alpha_{j}$ et $\kappa=1 /(3 g \alpha+1)$. Nous répartissons les entiers $n$ comptés dans $(3 \cdot 22)$ en deux classes, selon que l'on a ou non

$$
\prod_{\substack{p \mid F(n) \\ p \leqslant v}} p \geqslant w^{\kappa}
$$

Le cas de la première classe relève du lemme 3.6 avec $h_{j} \equiv 1(1 \leqslant j \leqslant r)$. Son 
cardinal est

$$
\ll x \exp \left\{-\kappa \frac{\log w}{\log v}\right\},
$$

l'estimation étant trivialement satisfaite si $v \geqslant w^{\kappa}$.

Lorsque (3.23) n'a pas lieu, on peut écrire

$$
\sum_{\substack{\nu \\ p^{\| F(n)} \\ p \leqslant v}}(\nu-3 g \alpha) \log p>\log w-3 g \alpha \kappa \log w=\kappa \log w .
$$

Cela implique

$$
\prod_{\substack{p^{\nu} \| F(n) \\ \nu \geqslant 3 g \alpha+1}} p^{\nu}>w^{\kappa}
$$

Notons $t$ un entier générique tel que $p^{\nu} \| t \Rightarrow \nu \geqslant 3 g$. L'inégalité (3·24) implique l'existence d'un diviseur $t$ de $F^{*}(n)$ satisfaisant à $t>w^{\kappa / \alpha}$. Le cardinal de la seconde classe est donc au plus égal à

$$
\sum_{w^{\kappa / \alpha}<t \leqslant c_{9} x^{g}} \varrho^{*}(t)\left(\left[\frac{x}{t}\right]+1\right) \ll \sum_{t \leqslant c_{9} x^{g}} c_{10}^{\omega(t)}\left\{\frac{x}{t}\left(t w^{-\kappa / \alpha}\right)^{\eta}+\left(\frac{x^{g}}{t}\right)^{1-\eta}\right\}
$$

où $\eta$ est un paramètre arbitraire $(0 \leqslant \eta<1)$ et où nous avons utilisé la majoration $\varrho^{*}(t) \leqslant c_{10}^{\omega(t)}$ qui découle de $(2 \cdot 14)$. La série

$$
\sum_{t} c_{10}^{\omega(t)} t^{\eta-1}
$$

est convergente pour tout $\eta<1-1 / 3 g$. En choisissant par exemple $\eta=1-1 / 2 g$, nous obtenons la majoration

$$
\ll x w^{-\kappa / 2 \alpha g}+\sqrt{x} .
$$

Cela suffit pleinement à compléter la démonstration.

Le lemme suivant montre que, lorsque $d=1$, l'estimation (3.9) du lemme 3.5 fournit l'ordre de grandeur exact de la somme considérée si les $h_{j}(p)$ sont minorés par une constante positive. Ce résultat est analogue au Satz 2 de Wolke [19]. 
Lemme 3.8. Soient $A^{\prime}, A^{\prime \prime}$ des constantes positives et $h_{1}, h_{2}, \ldots, h_{r}$ des fonctions arithmétiques fortement multiplicatives satisfaisant pour tout nombre premier $p$ à

$$
A^{\prime} \leqslant h_{j}(p) \leqslant A^{\prime \prime} \quad(1 \leqslant j \leqslant r) .
$$

Alors on a pour $x \geqslant 1$

$$
\sum_{n \leqslant x} \prod_{j=1}^{r} h_{j}(F(n)) \asymp x \exp \left\{\sum_{j=1}^{r} \sum_{p \leqslant x} \frac{\varrho_{j}(p)\left(h_{j}(p)-1\right)}{p}\right\}
$$

Démonstration. La majoration découle de (3.9) avec $d=1$. Pour établir la minoration, nous restreignons la somme en $n$ aux entiers qui satisfont les conditions suivantes

$$
\left\{\begin{array}{l}
F_{j}(n)=D_{j} A_{j} B_{j} \quad(1 \leqslant j \leqslant r) \\
D_{j} \mid D^{\infty} \\
\left(A_{j}, D\right)=1, A_{j} \leqslant X:=x^{c_{6} / 2 r}, P^{-}\left(B_{j}\right)>X .
\end{array}\right.
$$

où $c_{6}=c_{6}\left(\frac{1}{2}, F\right)$ est la constante du lemme 3.4. Notant $d_{j}, a_{j}, b_{j}$ les noyaux sans facteur carré respectifs de $D_{j}, A_{j}, B_{j}$ on a alors

$$
h_{j}(F(n))=h_{j}\left(d_{j}\right) h_{j}\left(a_{j}\right) h_{j}\left(b_{j}\right) \gg h_{j}\left(a_{j}\right)
$$

puisque

$$
h_{j}\left(d_{j}\right) \geqslant \min \left(1, A^{\prime}\right)^{\omega(D)}
$$

et

$$
h_{j}\left(b_{j}\right) \geqslant \min \left(1, A^{\prime}\right)^{\left(2 r g / c_{6}\right)+1} \quad\left(x \geqslant x_{0}\right) .
$$

La somme en $n$ de $(3 \cdot 25)$ est donc

$$
\begin{aligned}
& \gg \sum_{\substack{\left.a_{1}, \ldots, a_{r} \leqslant X \\
\left(a_{j}, D\right)=1 \leqslant j \leqslant r\right)}}^{\prime} \prod_{\substack{j=1 \\
(1 \leqslant j}}^{r} h_{j}\left(a_{j}\right) \quad \sum_{\substack{n \leqslant x \\
a_{j}\left|F_{j}(n)(1 \leqslant j \leqslant r) \\
p\right| F(n) \Rightarrow p \mid D \prod_{j=1}^{r} a_{j} \text { ou } p>X}}^{r} 1 \\
& \gg \frac{x}{(\log x)^{r}} \sum_{\substack{a_{1}, \ldots, a_{r} \leqslant X \\
\left(a_{j}, D a_{j}\right)=1 \\
(1 \leqslant i<j \leqslant r)}}^{\prime} \prod_{j=1}^{r} \frac{\varrho_{j}\left(a_{j}\right) h_{j}\left(a_{j}\right)}{\varphi^{*}\left(a_{j}\right)} \geqslant \frac{x}{(\log x)^{r}} \sum_{\substack{a \leqslant X \\
(a, D)=1}}^{\prime} \frac{\vartheta(a)}{a},
\end{aligned}
$$

d'après le lemme 3.4 , où $\vartheta$ est la fonction multiplicative définie sur les entiers sans facteur carré par $\vartheta(p)=\left(1-\varrho^{*}(p) / p\right)^{-1} \sum_{j=1}^{r} \varrho_{j}(p) h_{j}(p)$. Il nous suffit donc maintenant de montrer que l'on a

$$
\sum_{\substack{a \leqslant X \\(a, D)=1}}^{\prime} \frac{\vartheta(a)}{a} \gg \exp \left\{\sum_{p \leqslant x} \frac{\vartheta(p)}{p}\right\} .
$$


Soit $\varepsilon>0$, et $Z:=X^{\varepsilon}$. Le membre de gauche de (3·26) est au moins égal à

$$
\sum_{\substack{P^{+}(a) \leqslant Z \\(a, D)=1}}^{\prime} \frac{\vartheta(a)}{a}-\sum_{\substack{P^{+}(a) \leqslant Z \\ a>X \\(a, D)=1}}^{\prime} \frac{\vartheta(a)}{a} .
$$

La première de ces deux sommes vaut

$$
\prod_{\substack{p \leqslant Z \\ p \nmid D}}\left(1+\frac{\vartheta(p)}{p}\right) \gg \exp \left\{\sum_{p \leqslant Z} \frac{\vartheta(p)}{p}\right\}
$$

puisque $\vartheta(p) \ll 1$. La seconde est majorée pour tout $\eta>0$ par

$$
X^{-\eta} \prod_{p \leqslant Z}\left(1+\frac{\vartheta(p)}{p^{1-\eta}}\right) \leqslant \exp \left\{-\frac{\eta}{\varepsilon} \log Z+\sum_{p \leqslant Z} \frac{\vartheta(p)}{p^{1-\eta}}\right\} .
$$

Pour le choix $\eta=1 / \log Z$, cette expression ne dépasse pas

$$
\exp \left\{-\frac{1}{\varepsilon}+\sum_{p \leqslant Z} \frac{\vartheta(p)}{p}+O\left(\frac{1}{\log Z} \sum_{p \leqslant Z} \frac{\log p}{p}\right)\right\}
$$

où le terme d'erreur est borné indépendamment de $\varepsilon$. Lorsque $\varepsilon$ est assez petit, $(3 \cdot 28)$ est donc au plus égal à la moitié de $(3 \cdot 27)$. Cela établit bien $(3 \cdot 26)$ et achève la démonstration.

Lemme 3.9. Soit $g(d)$ la fonction arithmétique multiplicative définie au lemme 3.5. On suppose de plus l'existence de nombres réels $\mu_{j} \geqslant 0(1 \leqslant j \leqslant r)$ tels que l'on ait

$$
h_{j}(p)=\mu_{j} \quad(1 \leqslant j \leqslant r)
$$

sauf au plus pour un nombre fini de nombres premiers $p$. Alors, pour chaque entier $N \geqslant 1$, il existe un polynôme $P_{N}(t)$, de degré $N$, et dont les coefficients ne dépendent que des $h_{j}$ et de $F$, tel que l'on ait pour $x$ infini

$$
\sum_{d \leqslant x} g(d)=x(\log x)^{\mu-1}\left\{P_{N}\left(\frac{1}{\log x}\right)+O_{N}\left(\left(\frac{1}{\log x}\right)^{N+1}\right)\right\}
$$

avec

$$
\mu:=\sum_{j=1}^{r} \alpha_{j} \mu_{j}
$$


Démonstration. Soit $s=\sigma+i \tau$ un nombre complexe de partie réelle $\sigma>1$. Pour tous les $p$ sauf un nombre fini, on a, par $(3 \cdot 10)$,

$$
\sum_{\nu=0}^{\infty} g\left(p^{\nu}\right) p^{-\nu s}=1+\left(1-\frac{\varrho^{*}(p)}{p}\right) \sum_{j=1}^{r} \mu_{j} \varrho_{j}(p) \sum_{\nu=0}^{\infty} p^{\nu(1-s)-\left\lceil\nu / \alpha_{j}\right\rceil} .
$$

La somme en $\nu$ vaut

$$
\sum_{m=1}^{\infty} \sum_{(m-1) \alpha_{j}<\nu \leqslant m \alpha_{j}} p^{\nu(1-s)-m}=\sum_{\nu=1}^{\alpha_{j}} p^{-1-\nu(s-1)}+R_{p}(s),
$$

où $R_{p}(s)$ est une fonction holomorphe de $s$ pour $\sigma>1-1 / 2 \alpha_{j}$ et satisfait dans ce domaine à la majoration

$$
R_{p}(s) \ll p^{2 \alpha_{j}(1-\sigma)-2}\left(1+p^{\left(\alpha_{j}-1\right)(\sigma-1)}\right)
$$

Pour $\sigma \geqslant \sigma_{0}:=1-1 /\left(4 \max _{1 \leqslant j \leqslant r} \alpha_{j}\right)$ et $p$ assez grand, on peut donc écrire

$$
\sum_{\nu=0}^{\infty} g\left(p^{\nu}\right) p^{-\nu s}=\prod_{j=1}^{r} \prod_{\nu=1}^{\alpha_{j}}\left(1-p^{-1-\nu(s-1)}\right)^{-\mu_{j} \varrho_{j}(p)} \exp V_{p}(s)
$$

où $V_{p}(s)$ est une fonction holomorphe en $s$ dans le demi-plan $\sigma \geqslant \sigma_{0}$ qui satisfait dans la même région à

$$
V_{p}(s) \ll p^{-3 / 2} .
$$

De plus, lorsque $p$ est borné les facteurs locaux

$$
\sum_{\nu=0}^{\infty} g\left(p^{\nu}\right) p^{-\nu s}
$$

définissent des fonctions holomorphes pour $\sigma \geqslant \sigma_{0}$. Cela découle immédiatement de (3.10) et $(2 \cdot 15)$ sous la forme

$$
g\left(p^{\nu}\right) \ll \sum_{j=1}^{r} p^{\nu-\left\lceil\nu / \alpha_{j}\right\rceil} .
$$

Nous pouvons donc écrire pour $\sigma>1$

$$
\sum_{d=1}^{\infty} g(d) d^{-s}=\prod_{j=1}^{r} \prod_{\nu=1}^{\alpha_{j}} \prod_{p}\left(1-p^{-1-\nu(s-1)}\right)^{-\mu_{j} \varrho_{j}(p)} \Phi(s),
$$

où $\Phi$ est une fonction holomorphe et bornée pour $\sigma \geqslant \sigma_{0}$, avec $\Phi(1) \neq 0$. 
Considérons maintenant la fonction zêta de Dedekind, $\zeta_{j}(s)$, du corps de nombres $\mathbb{Q}\left(\vartheta_{j}\right)$ engendré par un zéro $\vartheta_{j}$ de $F_{j}$. On peut écrire, pour $\sigma>1$, cette fonction comme un produit eulérien, soit

$$
\zeta_{j}(s)=\prod_{\wp}\left\{1-(N \wp)^{-s}\right\}^{-1}
$$

où $\wp$ parcourt l'ensemble des idéaux premiers de l'anneau des entiers de $\mathbb{Q}\left(\vartheta_{j}\right)$ et $N$ désigne la norme de $\mathbb{Q}\left(\vartheta_{j}\right)$ sur $\mathbb{Q}$. Pour chaque $\wp$, on a $N \wp=p^{f}$ ou $p$ est un nombre rationnel. Comme l'a montré Erdős dans [4], la partie du produit $(3 \cdot 33)$ correspondant aux $\wp$ tels que $f \geqslant 2$ ou $p \mid D$ définit une fonction holomorphe, bornée et sans zéro pour $\sigma \geqslant \sigma_{1}>\frac{1}{2}$. Pour tous les autres $\wp$, la valeur $p=N \wp$ apparaît exactement $\varrho_{j}(p)$ fois, de sorte que l'on peut écrire

$$
\prod_{p}\left(1-p^{-s}\right)^{-\varrho_{j}(p)}=\zeta_{j}(s) \Phi_{j}(s) \quad(\sigma>1)
$$

où $\Phi_{j}(s)$ est holomorphe, bornée, et sans zéro pour $\sigma \geqslant \sigma_{0}$. En reportant dans $(3 \cdot 32)$, il suit

$$
\sum_{d=1}^{\infty} g(d) d^{-s}=\prod_{j=1}^{r} \prod_{\nu=1}^{\alpha_{j}} \zeta_{j}(1+\nu(s-1))^{\mu_{j}} \Psi(s) \quad(\sigma>1)
$$

où $\Psi(s)$ est holomorphe et bornée pour $\sigma \geqslant \sigma_{0}$, avec $\Psi(1) \neq 0$. La série $(3 \cdot 35)$ possède donc en $s=1$ une singularité de type $(s-1)^{-\mu}$ et est prolongeable holomorphiquement dans une région $\left\{s: \sigma \geqslant 1-c /\left(1+\log ^{+}|\tau|\right)\right\} \backslash[1-c, 1]$ où $c$ est une constante positive convenable. Cela découle des propriétés classiques des fonctions zêta de Dedekind — cf. Landau [11], Satz 185, p. 105. De plus, le fait que les $\zeta_{j}(s)$ soient d'ordre fini dans toute bande verticale (cf [11] Satz 171, p. 87) implique, grâce à un théorème général concernant les séries de Dirichlet, que le prolongement $\mathcal{G}$ de $(3 \cdot 35)$ satisfait, pour tout $\varepsilon>0$, à

$$
\mathcal{G}(s) \ll_{\varepsilon}|\tau|^{\varepsilon} \quad(|\tau| \geqslant 1, \sigma \geqslant 1-c /(1+\log |\tau|)) .
$$

On peut alors évaluer le membre de gauche de (3.29) par la technique usuelle d'intégration complexe, faisant apparaître un contour de Hankel autour de $s=1$. Cette méthode a été employée par Selberg [16] dans le cas des puissances complexes de la fonction zêta de Riemann et fut développée par Delange dans les années soixante (voir en particulier [1]). Les calculs étant identiques mutatis mutandis, nous omettons les détails. 


\section{Répartition globale des diviseurs dans les suites polynomiales}

Cette section est dévolue à l'étude de diverses moyennes pondérées de fonctions liées à la répartition globale des diviseurs de $F(n)$.

Nous nous donnons un paramètre $L$ satisfaisant à

$$
0 \leqslant L \leqslant \log _{2} y
$$

et nous posons

$$
Y:=\exp \exp L \leqslant y .
$$

Pour chaque entier $n$ et tout $j(1 \leqslant j \leqslant r)$ nous introduisons les fonctions

$$
n_{j L}:=\prod_{\substack{p \mid F_{j}(n) \\ p \nmid D, p \leqslant Y}} p^{\alpha_{j}} \quad \text { et } \quad n_{L}:=\prod_{j=1}^{r} n_{j L} .
$$

D'après le choix de $D$ au paragraphe 2 , les $n_{j L}$ sont deux à deux premiers entre eux. On a identiquement

$$
\tau\left(n_{L}\right)=\prod_{j=1}^{r} H_{j}\left(F_{j}(n)\right),
$$

où $H_{j}$ est la fonction arithmétique fortement multiplicative définie par

$$
H_{j}(p)= \begin{cases}\alpha_{j}+1 & \text { si } p \nmid D, p \leqslant Y, \\ 1 & \text { si } p \mid D \text { ou } p>Y .\end{cases}
$$

Dans toute cette section, nous supposons que $\beta(y, z) \in[0, B]$. Nous nous donnons une constante positive arbitraire $v_{0}$ et considérons un nombre réel $v$ $\left(0 \leqslant v \leqslant v_{0}\right)$. Toutes les constantes, implicites ou explicites, peuvent dépendre de $B, v_{0}, F$ mais pas de $L$.

Lemme 4.1. Pour $x \geqslant 2,2 \leqslant y \leqslant x$ et $0 \leqslant v \leqslant v_{0}$, on a

$$
\sum_{n \leqslant x} \tau\left(n_{L}\right)^{v} \asymp x \mathrm{e}^{\gamma(v) L} .
$$

Démonstration. Cela découle du lemme 3.8 et de l'identité $(4 \cdot 1)$, en posant $h_{j}=H_{j}^{v}(1 \leqslant j \leqslant r)$. 
Lemme 4.2. Posons $W(t):=\exp \{t-2 \sqrt{t \log t}\}(t>1)$. Désignons, pour tous $v>0, \varepsilon>0$, par $\mathcal{A}(v, \varepsilon)$ l'ensemble des entiers $n$ tels que

$$
\min _{\varepsilon^{-1} \mathrm{e}^{-L} \leqslant|\vartheta| \leqslant 1} \tau_{\vartheta}\left(n_{L}\right)^{-1} W(L+\log |\vartheta|)^{-\gamma^{\prime}(v)} \leqslant \tau\left(n_{L}\right)^{-1} .
$$

Pour chaque $\varepsilon>0$, il existe une constante $y_{0}(\varepsilon)$ telle que l'on ait, lorsque $y_{0}(\varepsilon) \leqslant y \leqslant x$ et $0 \leqslant v \leqslant v_{0}$,

$$
\sum_{\substack{n \leqslant x \\ n \in \mathcal{A}(v, \varepsilon)}} \tau\left(n_{L}\right)^{v} \ll \frac{x \mathrm{e}^{\gamma(v) L}}{\log (1 / \varepsilon)} .
$$

Démonstration. Pour chaque $\vartheta\left(\varepsilon^{-1} \mathrm{e}^{-L} \leqslant|\vartheta| \leqslant 1\right)$, posons

$$
m(\vartheta)=L+[\log |\vartheta|]
$$

de sorte que $\log (1 / \varepsilon)-1<m(\vartheta) \leqslant L$. Si $n$ est compté dans $\mathcal{A}(v, \varepsilon)$ et si $\vartheta$ réalise le minimum $(4 \cdot 4)$, on a

$$
\begin{aligned}
1 & \leqslant \mathrm{e}^{\gamma^{\prime}(v)} W(m(\vartheta))^{\gamma^{\prime}(v)} \tau_{\vartheta}\left(n_{L}\right) \tau\left(n_{L}\right)^{-1} \\
& \leqslant \mathrm{e}^{\gamma^{\prime}(v)} W(m(\vartheta))^{\gamma^{\prime}(v)} \prod_{\substack{p^{\nu} \| n_{L} \\
L-m(\vartheta) \leqslant \log _{2} p \leqslant L}}(\nu+1)^{-1} .
\end{aligned}
$$

Soit $w=w(m(\vartheta))$ un paramètre tel que $0<w \ll 1$. Élevons l'inégalité précédente à la puissance $w$, multiplions les deux membres par $\tau\left(n_{L}\right)^{v}$ et sommons sur toutes les valeurs possibles de $m=m(\vartheta)$. Nous obtenons, pour $n$ dans $\mathcal{A}(v, \varepsilon)$,

$$
\tau\left(n_{L}\right)^{v} \ll \sum_{\log 1 / \varepsilon-1<m \leqslant L} W(m)^{w(m) \gamma^{\prime}(v)} \prod_{j=1}^{r} h_{j}\left(F_{j}(n) ; m\right)
$$

où $h_{j}(n ; m)$ est la fonction fortement multiplicative de $n$ définie par

$$
h_{j}(p ; m)= \begin{cases}\left(\alpha_{j}+1\right)^{v} & \text { si } p \nmid D, \log _{2} p \leqslant L-m, \\ \left(\alpha_{j}+1\right)^{v-w(m)} & \text { si } p \nmid D, L-m<\log _{2} p \leqslant L, \\ 1 & \text { si } p \mid D \text { ou } \log _{2} p>L .\end{cases}
$$


Sommons l'inégalité (4.6) pour $n \leqslant x$ et $n \in \mathcal{A}(v, \varepsilon)$. Après interversion de sommations, nous pouvons faire appel au lemme 3.5 pour évaluer la somme en $n$ dans le membre de droite. Nous obtenons que le membre de gauche de $(4.5)$ est

$$
\ll x \mathrm{e}^{\gamma(v) L} \sum_{\log (1 / \varepsilon)-1 \leqslant m \leqslant L} \mathrm{e}^{-m \Xi(m)}
$$

avec

$\Xi(m):=\sum_{j=1}^{r}\left(\alpha_{j}+1\right)^{v}\left\{1-\left(\alpha_{j}+1\right)^{-w(m)}-w(m)\left(1-2 \sqrt{\frac{\log m}{m}}\right) \log \left(\alpha_{j}+1\right)\right\}$

L'expression entre accolades est certainement positive lorsque

$$
1-2 \sqrt{\frac{\log m}{m}} \leqslant\left(\alpha_{j}+1\right)^{-w(m)}<1 .
$$

Nous choisissons

$$
w(m)=-\frac{\log (1-2 \sqrt{(\log m) / m})}{\log \max _{1 \leqslant j \leqslant r}\left(\alpha_{j}+1\right)} .
$$

On a alors

$$
\Xi(m) \geqslant Q\left(1-2 \sqrt{\frac{\log m}{m}}\right) \geqslant 2 \frac{\log m}{m} .
$$

En reportant dans $(4 \cdot 7)$, on obtient que la somme en $m$ ne dépasse pas

$$
\sum_{m \geqslant \log (1 / \varepsilon)-1} \frac{1}{m^{2}} \ll \frac{1}{\log (1 / \varepsilon)} .
$$

Cela achève la démonstration.

Lemme 4.3. Pour tous $v\left(0 \leqslant v \leqslant v_{0}\right)$, et $\psi \geqslant 0$, soit $\mathcal{B}(v, \psi)$ l'ensemble des entiers $n$ tels que

$$
\left|\log \tau\left(n_{L}\right)-\gamma^{\prime}(v) L\right|>\psi \gamma^{\prime}(v) \sqrt{L} .
$$

On a pour $x \geqslant 2,2 \leqslant y \leqslant x$ et $0 \leqslant \psi \leqslant \frac{1}{3} \sqrt{L}$,

$$
\sum_{\substack{n \leqslant x \\ n \in \mathcal{B}(v, \psi)}} \tau\left(n_{L}\right)^{v} \ll \mathrm{e}^{-\frac{1}{3} \psi^{2}} x \mathrm{e}^{\gamma(v) L} .
$$


Démonstration. Nous procédons de manière analogue à la preuve du lemme précédent, mais les détails sont ici plus simples car la situation correspond à $\vartheta=1$. Contentons-nous d'estimer par exemple la contribution $R$ au membre de gauche de (4.9) des entiers $n$ tels que

$$
\tau\left(n_{L}\right)>\mathrm{e}^{\gamma^{\prime}(v)\{L+\psi \sqrt{L}\}} .
$$

Le cas des autres exceptions relève d'une manipulation symétrique.

Pour chaque valeur du paramètre $w \geqslant 0$, on a

$$
\begin{aligned}
R & \leqslant \sum_{n \leqslant x} \tau\left(n_{L}\right)^{v+w} \mathrm{e}^{-w \gamma^{\prime}(v)\{L+\psi \sqrt{L}\}} \\
& \ll x \mathrm{e}^{(\gamma(v)-X) L},
\end{aligned}
$$

d'après le lemme 4.1, avec

$$
X:=\sum_{j=1}^{r}\left(\alpha_{j}+1\right)^{v}\left\{1-\left(\alpha_{j}+1\right)^{w}+\left(1+\frac{\psi}{\sqrt{L}}\right) w \log \left(\alpha_{j}+1\right)\right\}
$$

Comme précédemment, on a

$$
X \geqslant Q\left(1+\frac{\psi}{\sqrt{L}}\right) \geqslant \frac{\psi^{2}}{3 L}
$$

pour le choix

$$
w=\frac{\log (1+\psi / \sqrt{L})}{\log \max \left(\alpha_{j}+1\right)} .
$$

Cela implique bien l'estimation annoncée.

Lemme 4.4. Posons $\kappa=\kappa(v, F)=2 \sum_{j=1}^{r} \alpha_{j}\left(\alpha_{j}+1\right)^{v}$. On a pour $x \geqslant 2$, $2 \leqslant y \leqslant x, 0 \leqslant v \leqslant v_{0}$ et $\vartheta \in \mathbb{R}$,

$$
\sum_{n \leqslant x} \tau\left(n_{L}\right)^{v-1} \tau_{\vartheta}\left(n_{L}\right)^{-1}\left|\tau\left(n_{L}, \vartheta\right)\right|^{2} \ll x(\log (3+|\vartheta|))^{\kappa} \mathrm{e}^{\gamma(v) L} .
$$


Démonstration. Lorsque $|\vartheta| \leqslant \mathrm{e}^{-L}$, on a $\tau_{\vartheta}\left(n_{L}\right)=\tau\left(n_{L}\right)$ pour tout $n$ et la majoration triviale

$$
\left|\tau\left(n_{L}, \vartheta\right)\right| \leqslant \tau\left(n_{L}\right)
$$

fournit l'estimation souhaitée, compte tenu de (4·3).

Supposons désormais $|\vartheta|>\mathrm{e}^{-L}$. La somme à majorer, disons $S(\vartheta)$, peut encore s'écrire

$$
S(\vartheta)=\sum_{n \leqslant x} \prod_{j=1}^{r} g_{j}\left(F_{j}(n)\right),
$$

où $g_{j}(1 \leqslant j \leqslant r)$ est la fonction arithmétique fortement multiplicative définie sur les nombres premiers par

$$
g_{j}(p)= \begin{cases}\left(\alpha_{j}+1\right)^{v-2}\left|\sum_{\nu=0}^{\alpha_{j}} p^{i \nu \vartheta}\right|^{2} & \text { si } p \nmid D, \log p \leqslant 1 /|\vartheta|, \\ \left(\alpha_{j}+1\right)^{v-1}\left|\sum_{\nu=0}^{\alpha_{j}} p^{i \nu \vartheta}\right|^{2} & \text { si } p \nmid D, 1 /|\vartheta|<\log p \leqslant \mathrm{e}^{L}, \\ 1 & \text { si } p \mid D \text { ou } \log p>\mathrm{e}^{L} .\end{cases}
$$

Nous pouvons donc faire appel au lemme 3.5.

Lorsque $|\vartheta| \leqslant 2$, nous majorons trivialement $g_{j}(p)$ par $\left(\alpha_{j}+1\right)^{v}$ lorsque $\log p \leqslant 1 /|\vartheta|$. Nous obtenons

$$
\begin{aligned}
S(\vartheta) \ll & x \exp \left\{\sum _ { j = 1 } ^ { r } \left(\sum_{|\vartheta| \log p \leqslant 1}\left(\alpha_{j}+1\right)^{v} \frac{\varrho_{j}(p)}{p}\right.\right. \\
& \left.\left.+\sum_{1 /|\vartheta|<\log p \leqslant \mathrm{e}^{L}}\left(\alpha_{j}+1\right)^{v-1}\left|\sum_{\nu=0}^{\alpha_{j}} p^{i \nu \vartheta}\right|^{2} \frac{\varrho_{j}(p)}{p}-L\right)\right\} .
\end{aligned}
$$

Estimons la première somme en $p$ par le lemme 3.1, et la seconde par le lemme 3.3 avec

$$
f(t)=\left(\alpha_{j}+1\right)^{v-1}\left|\sum_{0 \leqslant \nu \leqslant \alpha_{j}} \mathrm{e}^{i \nu t}\right|^{2}
$$

de moyenne $\bar{f}=\left(\alpha_{j}+1\right)^{v}$. Nous obtenons

$$
S(\vartheta) \ll x \mathrm{e}^{\gamma(v) L},
$$

ce qui est bien en accord avec (4·11). 
Lorsque $|\vartheta|>2$, on a $\tau_{\vartheta}\left(n_{L}\right)=1$ pour tout $n$, et le lemme 3.5 fournit la borne

$$
S(\vartheta) \ll x \exp \left\{\sum_{j=1}^{r} \sum_{p \leqslant Y}\left(\left(\alpha_{j}+1\right)^{v-1}\left|\sum_{\nu=0}^{\alpha_{j}} p^{i \nu \vartheta}\right|^{2}-1\right) \frac{\varrho_{j}(p)}{p}\right\}
$$

On applique le lemme 3.3 à la sous-somme en $p$ correspondant à l'intervalle de sommation (éventuellement vide)

$$
c^{-2}\{\log (3+|\vartheta|)\}^{2}<\log p \leqslant \mathrm{e}^{L}
$$

où $c$ est une constante positive minorant tous les $c\left(F_{j}\right)$ tels qu'ils sont définis au lemme 3.3. On obtient une contribution ne dépassant pas

$$
\left\{\left(\alpha_{j}+1\right)^{v}-1\right\}\left\{L-2 \log _{2}(3+|\vartheta|)\right\}+O(1) .
$$

La sous-somme complémentaire est majorée trivialement, en estimant simplement $\left|\sum p^{i \nu \vartheta}\right|$ par $\alpha_{j}+1$. Elle est au plus égale à

$$
2\left(\left(\alpha_{j}+1\right)^{v+1}-1\right) \log _{2}(3+|\vartheta|)+O(1) .
$$

En regroupant ces évaluations, on obtient bien le résultat indiqué.

\section{Majorations de $H_{F}(x, y, z)$}

Nous nous proposons dans cette section d'établir les bornes supérieures des encadrements $(1 \cdot 13)$ et $(1 \cdot 14)$ de notre théorème.

Commençons par (1.13). Nous allons montrer que, pour une proportion positive des entiers $n \leqslant x$, minorée indépendamment de $y$, le nombre $F(n)$ ne possède aucun diviseur dans $] y, 2 y]$. Comme $H_{F}(x, y, z)$ est une fonction croissante de $z$ (et donc décroissante de $\beta$ ), cela suffit pleinement à prouver l'estimation supérieure de (1·13).

Considérons la décomposition canonique

$$
F(n)=a_{n} b_{n} \quad\left(P^{+}\left(a_{n}\right) \leqslant 2 y<P^{-}\left(b_{n}\right)\right) .
$$

On a certainement $\tau(F(n) ; y, 2 y)=0$ lorsque $a_{n} \leqslant y$. Pour chaque $\varepsilon>0$, on peut donc écrire

$$
x-H_{F}(x, y, 2 y) \geqslant \sum_{\substack{n \leqslant x \\ a_{n} \leqslant y}} 1 \sum_{\substack{n \leqslant x \\ P^{+}\left(a_{n}\right) \leqslant y^{\varepsilon}}} 1-\sum_{\substack{n \leqslant x \\ P^{+}\left(a_{n}\right) \leqslant y^{\varepsilon} \\ a_{n}>y}} 1 .
$$

Le premier terme de cette minoration est égal au nombre des entiers $n \leqslant x$ tels que $F^{*}(n)$ ne possède aucun facteur premier dans $\left.] y^{\varepsilon}, 2 y\right]$. D'après le lemme fondamental de la théorie du crible (cf. par exemple [7], theorem 7.1), il est

$$
\asymp \varepsilon^{r} x
$$


sous l'hypothèse $y_{0} \leqslant y \leqslant x^{c_{0}}$, avec $y_{0}=y_{0}(\varepsilon, F)$ et $c_{0}=c_{0}(F)$ convenables. Or, le lemme 3.7 nous permet de majorer le second cardinal par

$$
\ll \mathrm{e}^{-c_{8} / \varepsilon} x
$$

Pour $\varepsilon$ assez petit mais fixé, nous obtenons bien l'estimation souhaitée.

Établissons maintenant la majoration de (1·14), c'est-à-dire l'estimation

$$
H_{F}(x, y, z) \ll x(\log y)^{-\delta(\beta, F)}
$$

sous les conditions

$$
y_{0} \leqslant y \leqslant x^{1-K}, \quad \max \left(0, \log \widehat{\tau}(F)-1-\frac{c_{1}}{\sqrt{\log _{2} y}}\right) \leqslant \beta \leqslant B .
$$

Nous pouvons en fait supposer que $\beta \geqslant \log \widehat{\tau}(F)-1$ puisque $\delta(\beta, F)=0$ dans le cas contraire. En particulier, on a donc

$$
\gamma^{\prime}(u)=\beta+1 .
$$

Nous distinguons deux cas, selon que $u \leqslant 1$ ou non. Dans le premier cas, soit $\beta \leqslant \gamma^{\prime}(1)-1$, nous écrivons

$$
H_{F}(x, y, z) \leqslant H_{1}+H_{2}
$$

avec

$$
H_{1}:=\sum_{\substack{n \leqslant x \\ \tau\left(n_{L}\right)>(\log y)^{1+\beta}}} 1, \quad H_{2}:=\sum_{\substack{n \leqslant x \\ \tau\left(n_{L}\right) \leqslant(\log y)^{1+\beta}}} \tau(F(n) ; y, z)
$$

pour le choix (valable dans cette section uniquement)

$$
L=\log _{2} y .
$$

Le lemme 4.1 avec $v=u$ fournit

$$
H_{1} \leqslant \sum_{n \leqslant x} \tau\left(n_{L}\right)^{u}(\log y)^{-u(1+\beta)} \ll x(\log y)^{\gamma(u)-u(1+\beta)} .
$$

Grâce à (5.2), cela équivaut bien à (5·1). Pour estimer $H_{2}$, donnons-nous un paramètre $v \geqslant 0$. On a

$$
\begin{aligned}
H_{2} & \leqslant(\log y)^{v \gamma^{\prime}(u)} \sum_{n \leqslant x} \tau(F(n) ; y, z) \tau\left(n_{L}\right)^{-v} \\
& =(\log y)^{v \gamma^{\prime}(u)} \sum_{y<d \leqslant z} \sum_{\substack{n \leqslant x \\
d \mid F(n)}} \tau\left(n_{L}\right)^{-v} .
\end{aligned}
$$

Puisque $d \leqslant 2 y \leqslant 2 x^{1-K}$, on peut appliquer le lemme 3.5 pour majorer la 
somme intérieure, compte tenu de l'identité (4·1). On obtient la majoration

$$
\ll x \frac{g(d)}{d}(\log y)^{\gamma(-v)}
$$

où $g$ est définie par $(3 \cdot 10)$ avec $h_{j}(p)=\left(\alpha_{j}+1\right)^{-v}(p \nmid D, p \leqslant y)$. Par sommation d'Abel, le lemme 3.9 nous permet donc de montrer que

$$
\sum_{y<d \leqslant z} \frac{g(d)}{d} \ll \frac{z-y}{y}(\log y)^{\mu-1}
$$

avec

$$
\mu=\sum_{j=1}^{r} \alpha_{j}\left(\alpha_{j}+1\right)^{-v}=\gamma(1-v)-\gamma(-v) .
$$

Nous obtenons ainsi

$$
H_{2} \ll x(\log y)^{v \gamma^{\prime}(u)+\gamma(1-v)-\beta-1} .
$$

Pour le choix $v=1-u$ (qui est licite car $u \leqslant 1$ ) l'exposant de $\log y$ vaut $u \gamma^{\prime}(u)-\gamma(u)=-\delta(\beta, F)$. Cela établit encore (5.1) lorsque $\gamma(0)-1 \leqslant \beta \leqslant$ $\gamma(1)-1$.

Lorsque $\beta>\gamma(1)-1$, nous nous contentons de la majoration triviale

$$
H_{F}(x, y, z) \leqslant \sum_{n \leqslant x} \tau(F(n) ; y, z)=\sum_{y<d \leqslant z} \sum_{\substack{n \leqslant x \\ d \mid F(n)}} 1 \ll x \sum_{y<d \leqslant z} \frac{\varrho(d)}{d} .
$$

Nous pouvons de nouveau faire appel au lemme 3.9 pour estimer la somme en $d$ puisque $(2 \cdot 11)$ et $(3 \cdot 10)$ montrent que $\varrho(d)$ est majorée par la fonction $g(d)$ correspondant au cas $h_{j} \equiv 1(1 \leqslant j \leqslant r)$. On obtient comme annoncé

$$
H_{F}(x, y, z) \ll x\left(\frac{z-y}{y}\right)(\log y)^{\widehat{\Omega}(F)-1}=x(\log y)^{\widehat{\Omega}(F)-\beta-1} .
$$

Cela achève la preuve de $(5 \cdot 1)$.

\section{Minorations de $H_{F}(x, y, z)$}

Nous allons maintenant établir la validité des bornes inférieures de notre théorème. À cet effet, nous adoptons une méthode analogue à celle du chapitre 5 de [8] reposant sur l'idée que les quantités $\log d(d \mid F(n))$ sont bien réparties dans $[0, \log F(n)]$ dès qu'elles sont suffisamment nombreuses. 
Pour chaque entier $m \geqslant 1$, nous introduisons la fonction croissante

$$
\mathcal{F}(m ; t):=\tau\left(m ; 0, \mathrm{e}^{t}\right)=\operatorname{card}\left\{d: d \mid m, d \leqslant \mathrm{e}^{t}\right\} .
$$

La transformée de Fourier-Stieltjes est évidemment

$$
\tau(m, \vartheta)=\int_{-\infty}^{+\infty} \mathrm{e}^{i \vartheta t} \mathrm{~d} \mathcal{F}(m ; t)=\sum_{d \mid m} d^{i \vartheta} .
$$

Soit $\eta:=\log (z / y)$. Nous allons montrer que, relativement à la mesure discrète sur $\{n: n \leqslant x\}$ associée au poids $\tau\left(n_{L}\right)^{v}$, la fonction d'accroissement

$$
\Delta\left(n_{L} ; t\right):=\mathcal{F}\left(n_{L} ; t+\eta\right)-\mathcal{F}\left(n_{L} ; t\right)
$$

est «souvent» positive lorsque $v$ est sensiblement plus grand que $u$. (Nous choisirons $L \approx \log _{2} y-T$ où $T$ est une constante suffisamment grande.) Cela permet de mettre en évidence l'existence d'un ensemble assez riche de nombres premiers $p>\exp \exp L$ tels que $p n_{L}$ possède un diviseur dans $\left.] y, z\right]$. Un argument de crible permet ensuite de conclure.

Soit $\lambda(m)$ la mesure de Lebesgue de l'ensemble $\mathcal{L}(m)$ des nombres réels $t$ tels que $\Delta(m ; t)>0$. Commençons par établir une minoration pour $\lambda(m)$.

Lemme 6.1. Pour chaque entier $m \geqslant 1$, on a

$$
\tau(m)^{2} \leqslant 3 \pi \lambda(m) \int_{|\vartheta \eta| \leqslant 1}|\tau(m, \vartheta)|^{2} \mathrm{~d} \vartheta .
$$

Démonstration. Par l'inégalité de Cauchy-Schwarz, on peut écrire

$$
\eta^{2} \tau(m)^{2}=\left(\int_{-\infty}^{+\infty} \Delta(m ; t) \mathrm{d} t\right)^{2} \leqslant \lambda(m) \int_{-\infty}^{+\infty} \Delta(m ; t)^{2} \mathrm{~d} t .
$$

Posons

$$
w(t):=\left(\frac{\sin \frac{1}{2} t}{\frac{1}{2} t}\right)^{2}=\int_{-1}^{1}(1-|\vartheta|) \mathrm{e}^{i \vartheta t} \mathrm{~d} \vartheta .
$$

Alors pour tout $t \in \mathbb{R}$, on a

$$
\begin{aligned}
\Delta(m ; t) & \leqslant \frac{1}{w(1)} \sum_{d \mid m} w((\log d-t) / \eta) \\
& =\frac{\eta}{w(1)} \int_{|\vartheta \eta| \leqslant 1}(1-|\vartheta \eta|) \mathrm{e}^{i \vartheta t} \tau(m, \vartheta) \mathrm{d} \vartheta
\end{aligned}
$$

La formule de Parseval implique donc 


$$
\int_{-\infty}^{+\infty} \Delta(m ; t)^{2} \mathrm{~d} t \leqslant 2 \pi w(1)^{-2} \eta^{2} \int_{|\vartheta \eta| \leqslant 1}(1-|\vartheta \eta|)^{2}|\tau(m, \vartheta)|^{2} \mathrm{~d} \vartheta,
$$

d'où le résultat indiqué puisque $w(1)^{2}>2 / 3$.

Le résultat suivant constitue le point-clef de la démonstration.

Lemme 6.2. Soient $\varepsilon$ et $\beta_{0}$, des nombres réels positifs. Il existe des constantes $c_{11}=c_{11}(\varepsilon, B, F)>0$ et $y_{0}=y_{0}\left(\varepsilon, \beta_{0}, B, F\right)$ telles que l'on ait pour $y_{0} \leqslant y \leqslant x, \log _{2} y-\sqrt{\log _{2} y} \leqslant L \leqslant \log _{2} y, 0 \leqslant v \leqslant 1, \gamma^{\prime}(v) \geqslant 1$,

$$
\sum_{\substack{n \leqslant x \\ \lambda\left(n_{L}\right) \leqslant \varepsilon e^{\sigma L}}} \tau\left(n_{L}\right)^{v} \ll \frac{x \mathrm{e}^{\gamma(v) L}}{\log (1 / \varepsilon)},
$$

où l'on a posé

$$
\sigma=\sigma(\beta, v)=\left\{\begin{array}{lr}
\min \left\{1-c_{11} \sqrt{\frac{\log L}{L}}, \gamma^{\prime}(v)-\beta-\frac{c_{11}}{\sqrt{L}}\right\} & \text { si } 0 \leqslant \beta<\beta_{0}, \\
\min \left\{1, \gamma^{\prime}(v)-\beta-\frac{c_{11}}{\sqrt{L}}\right\} & \text { si } \beta_{0} \leqslant \beta \leqslant B .
\end{array}\right.
$$

Remarque. On a $\gamma^{\prime}(0)=\log \widehat{\tau}(F)$. Donc $\gamma^{\prime}(v) \geqslant \log 3>1$ dès que $F$ est irréductible.

Démonstration. D'après (6-1) la condition $\lambda\left(n_{L}\right) \leqslant \varepsilon \mathrm{e}^{\sigma L}$ implique

$$
3 \pi \varepsilon \mathrm{e}^{\sigma L} \int_{|\vartheta \eta| \leqslant 1} \frac{\left|\tau\left(n_{L}, \vartheta\right)\right|^{2}}{\tau\left(n_{L}\right)^{2}} \mathrm{~d} \vartheta \geqslant 1
$$

Puisque la contribution au membre de gauche des nombres réels $\vartheta$ tels que $12 \pi \varepsilon \mathrm{e}^{\sigma L}|\vartheta| \leqslant 1$ est trivialement $\leqslant \frac{1}{2}$, on voit que les entiers $n$ comptés dans $(6 \cdot 2)$ satisfont nécessairement

$$
\varepsilon_{1} \mathrm{e}^{\sigma L} \int_{1 /\left(\varepsilon_{1} \mathrm{e}^{\sigma L}\right)}^{1 / \eta} \frac{\left|\tau\left(n_{L}, \vartheta\right)\right|^{2}}{\tau\left(n_{L}\right)^{2}} \mathrm{~d} \vartheta \geqslant 1
$$

où, pour simplifier l'écriture, nous avons posé $\varepsilon_{1}=12 \pi \varepsilon$. Appliquons maintenant les lemmes 4.2 et 4.3. Quitte à négliger un ensemble d'entiers exceptionnels dont la contribution à (6.2) est acceptable, cela nous permet de supposer que l'on a

$$
\tau\left(n_{L}\right)^{-1} \leqslant \tau_{\vartheta}\left(n_{L}\right)^{-1} W(L+\log \vartheta)^{-\gamma^{\prime}(v)} \quad\left(\varepsilon_{1}^{-1} \mathrm{e}^{-\sigma L} \leqslant \vartheta \leqslant 1\right)
$$

et

$$
\tau\left(n_{L}\right)^{-1} \leqslant \mathrm{e}^{-\gamma^{\prime}(v)\{L-\psi \sqrt{L}\}},
$$


où $W(t)$ est la fonction définie au lemme 4.2 et où nous avons posé

$$
\psi=\psi(\varepsilon)=\sqrt{3 \log _{2}(1 / \varepsilon)} .
$$

Reportons ces estimations dans $(6 \cdot 4)$ en utilisant (6.5) lorsque $\vartheta \leqslant 1$. Nous obtenons

$$
\begin{array}{r}
\varepsilon_{1} \mathrm{e}^{\sigma L}\left\{\int_{1 /\left(\varepsilon_{1} \mathrm{e}^{\sigma L}\right)}^{1} \frac{\left|\tau\left(n_{L}, \vartheta\right)\right|^{2} \mathrm{~d} \vartheta}{\tau\left(n_{L}\right) \tau_{\vartheta}\left(n_{L}\right) W(L+\log \vartheta) \gamma^{\prime}(v)}\right. \\
\left.+\int_{1}^{1 / \eta} \frac{\left|\tau\left(n_{L}, \vartheta\right)\right|^{2} \mathrm{~d} \vartheta}{\mathrm{e}^{\gamma^{\prime}(v)(L-\psi \sqrt{L})} \tau\left(n_{L}\right)}\right\} \geqslant 1 .
\end{array}
$$

Multiplions les deux membres de cette inégalité par $\tau\left(n_{L}\right)^{v}$ et sommons sur tous les entiers $n \leqslant x$ satisfaisant (6·4), (6·5) et (6·6). Grâce au lemme 4.4, nous obtenons ainsi que la contribution à $(6 \cdot 2)$ des entiers non exceptionnels est

$$
\ll \varepsilon x \mathrm{e}^{\gamma(v) L}\left\{I_{1}+I_{2}\right\}
$$

avec

$$
I_{1}:=\mathrm{e}^{\sigma L} \int_{1 / \varepsilon_{1} \mathrm{e}^{\sigma L}}^{1} \frac{\mathrm{d} \vartheta}{W(L+\log \vartheta)^{\gamma^{\prime}(v)}} \leqslant \mathrm{e}^{-(1-\sigma) L} \int_{1}^{L} \frac{\mathrm{e}^{t} \mathrm{~d} t}{W(t)^{\gamma^{\prime}(v)}}
$$

et

$$
I_{2}:=\mathrm{e}^{\left\{\sigma-\gamma^{\prime}(v)\right\} L+\psi \gamma^{\prime}(v) \sqrt{L}} \int_{1}^{1 / \eta}\{\log (3+\vartheta)\}^{\kappa} \mathrm{d} \vartheta .
$$

Nous allons montrer que $I_{1}$ et $I_{2}$ sont $O(1)$ pour le choix

$$
c_{11}:=2(\psi+2) \gamma^{\prime}(1)+12 B \text {. }
$$

Cela suffit pleinement à impliquer $(6 \cdot 2)$.

Majorons $I_{1}$. Lorsque $\beta<\beta_{0}$, on a $(1-\sigma) \geqslant c_{11} \sqrt{(\log L) / L}$, d'où

$$
I_{1} \leqslant \mathrm{e}^{-c_{11} \sqrt{L \log L}} \int_{1}^{L} \mathrm{e}^{\frac{1}{2} c_{11} \sqrt{t \log t}} \mathrm{~d} t \ll 1 .
$$

Lorsque $\beta \geqslant \beta_{0}$, nous distinguons deux cas. Si $\gamma^{\prime}(v) \geqslant 1+\frac{1}{2} \beta_{0}$, on a

$$
I_{1} \leqslant \int_{1}^{L} \mathrm{e}^{-\frac{1}{2} \beta_{0} t+2 \gamma^{\prime}(1) \sqrt{t \log t}} \mathrm{~d} t \ll 1 .
$$

Si $\gamma^{\prime}(v) \leqslant 1+\frac{1}{2} \beta_{0}$, alors $1-\sigma \geqslant 1-\gamma^{\prime}(v)+\beta \geqslant \frac{1}{2} \beta_{0}$, et il suit

$$
I_{1} \leqslant \mathrm{e}^{-\frac{1}{2} \beta_{0} L} \int_{1}^{L} \mathrm{e}^{2 \gamma^{\prime}(1) \sqrt{t \log t}} \mathrm{~d} t \ll 1
$$


Majorons $I_{2}$. Puisque $1 / \eta \leqslant \exp \{\beta L+2 B \sqrt{L}\} \leqslant \exp \left\{\beta L+\frac{1}{6} c_{11} \sqrt{L}\right\}$, on peut écrire

$$
I_{2} \leqslant \mathrm{e}^{-\left(\gamma^{\prime}(v)-\sigma-\beta\right) L+\frac{2}{3} c_{11} \sqrt{L}+O(\log L)}
$$

Or, on a en toute circonstance

$$
\left(\gamma^{\prime}(v)-\sigma-\beta\right) L \geqslant c_{11} \sqrt{L} .
$$

On voit donc que $I_{2}=O(1)$ lorsque $y_{0}$, et donc $L$, est assez grand. Cela achève la preuve du lemme 6.2.

Nous déduirons facilement les minorations annoncées pour $H_{F}(x, y, z)$ de la proposition suivante.

Proposition 6.3. Soient $K$ et $\beta_{0}$, des nombres réels de $] 0,1[$. Il existe des constantes positives $T, \varepsilon, c_{12}$ et $y_{0}$, ne dépendant que de $K, \beta_{0}, B$ et $F$, telles que l'on ait pour $y_{0} \leqslant y \leqslant x^{1-K}, 0 \leqslant \beta(y, z) \leqslant B, 0 \leqslant v \leqslant 1, \gamma^{\prime}(v) \geqslant 1$, et $\log _{2} y-2 T \leqslant L \leqslant \log _{2} y-T$,

$$
\sum_{n \leqslant x}^{(*)} \tau\left(n_{L}\right)^{v} \gg x(\log y)^{\gamma(v)+\sigma-1}
$$

où $\sigma=\sigma\left(\beta, v ; \beta_{0}, \varepsilon\right)$ est défini par $(6 \cdot 3)$ et où l'astérisque indique que la sommation est restreinte aux entiers $n$ tels que

$$
\text { (*) }\left\{\begin{array}{l}
\tau(F(n) ; y, z) \geqslant 1 \\
\left|\log \tau\left(n_{L}\right)-\gamma^{\prime}(v) L\right| \leqslant c_{12} \sqrt{L} .
\end{array}\right.
$$

Démonstration. Posons $R=\log (1 / \varepsilon), T=\log (4 R / K)$, et $\vartheta=\frac{1}{2} c_{6}\left(\frac{1}{2} K, F\right)$ où $c_{6}(K, F)$ est la quantité définie au lemme 3.4. Nous pouvons sans restreindre la généralité supposer que $T>\log (1 / \vartheta)$, d'où

$$
\exp \exp L \leqslant y^{\vartheta} \text {. }
$$

La première étape consiste à remarquer que l'on a pour $\varepsilon$ suffisamment petit mais fixé

$$
\sum_{n \leqslant x}^{(* *)} \tau\left(n_{L}\right)^{v} \asymp x \mathrm{e}^{\gamma(v) L}
$$

où la double astérisque indique que la variable de sommation $n$ est astreinte aux conditions

$$
(* *)\left\{\begin{array}{l}
\log n_{L} \leqslant R \mathrm{e}^{L} \\
\left|\log \tau\left(n_{L}\right)-\gamma^{\prime}(v) L\right| \leqslant R \sqrt{L}, \\
\lambda\left(n_{L}\right)>\varepsilon \mathrm{e}^{\sigma L}
\end{array}\right.
$$


On obtient (6.10) en appliquant le lemme 4.1 et en majorant les contributions respectives des entiers $n$ contrevenant à chacune des conditions $(* *)$ par les lemmes 3.6, 4.3 et 6.2 .

Ensuite, désignons par $\mathcal{M}$ l'ensemble des entiers $m$ qui sont de la forme $m=n_{L}$ pour au moins un $n$ compté dans (6-10). Alors le choix de $T$ implique pour tout $m$ de $\mathcal{M}$ et $y_{0}$ assez grand

$$
m \leqslant \exp \left\{R \mathrm{e}^{-T} \log y\right\} \leqslant \frac{1}{2} y^{K / 2} \leqslant \sqrt{y} .
$$

De plus, chaque $m$ de $\mathcal{M}$ possède une ou plusieurs représentations de la forme

$$
m=\prod_{j=1}^{r} m_{j}^{\alpha_{j}}
$$

où les $m_{j}$ sont sans facteur carré, deux à deux premiers entre eux, et premiers à $D$. Nous posons

$$
\chi(m):=\sum \prod_{j=1}^{r} \frac{\varrho_{j}\left(m_{j}\right)}{\varphi^{*}\left(m_{j}\right)} .
$$

où la sommation est étendue à toutes les représentations $(6 \cdot 12)$. Compte tenu de $(6 \cdot 9)$ et $(6 \cdot 11)$, il découle du lemme 3.4 que l'on a

$$
\sum_{n \leqslant x}^{(* *)} \tau\left(n_{L}\right)^{v} \asymp x \mathrm{e}^{-r L} \sum_{m \in \mathcal{M}} \chi(m) \tau(m)^{v}
$$

d'où, d'après $(6 \cdot 10)$,

$$
\sum_{m \in \mathcal{M}} \chi(m) \tau(m)^{v} \asymp \mathrm{e}^{\{\gamma(v)+r\} L .}
$$

Considérons maintenant la sous-somme $S$ de $(6 \cdot 10)$ restreinte aux entiers $n$ pour lesquels $F(n)$ possède un facteur premier $p$ tel que $\log y-\log p \in \mathcal{L}\left(n_{L}\right)$. Cette condition équivaut à l'existence d'un diviseur $d$ de $n_{L}$ tel que

$$
\log y-\log p<\log d \leqslant \log (z / y)+\log y-\log p
$$

c'est-à-dire

$$
y<p d \leqslant z .
$$

De plus, puisque $n_{L} \leqslant \sqrt{y}$, d'après le choix de $T$, on a $\sqrt{y}<p \leqslant 2 y$, donc $p \nmid d$ et $p d \mid F(n)$. Ainsi $S$ minore le membre de gauche de (6.8) lorsque $c_{12}=R$. 
Soit $V(n)$ le nombre de représentations de $F(n)$ sous la forme $F(n)=m p h$ avec les conditions

$$
\left\{\begin{array}{l}
m \in \mathcal{M} \\
\log p \in \log y-\mathcal{L}(m) \\
p^{\prime}\left|h \Rightarrow p^{\prime}\right| m p \text { ou } p^{\prime}>X:=\min \left(2 y, x^{\vartheta}\right)
\end{array}\right.
$$

Alors $n$ est certainement compté dans $S$ si $V(n) \neq 0$. De plus on a $V(n) \ll 1$ pour tout $n$. En effet, ou bien $2 y \leqslant x^{\vartheta}$, et le nombre $p$ et les facteurs premiers respectifs de $m$ et $h$ varient dans des intervalles disjoints - d'où $V(n) \leqslant 1$ ou bien $2 y>x^{\vartheta}$, et $V(n)$ n'excède pas le nombre total des facteurs premiers $>\sqrt{y}$ de $F(n)$ - d'où $V(n) \ll g / \vartheta \ll 1$. On peut donc écrire

$$
S \gg \sum_{n \leqslant x}^{(* *)} V(n) \tau\left(n_{L}\right)^{v} \gg \sum_{m \in \mathcal{M}} \tau(m)^{v} \sum_{\log p \in \log y-\mathcal{L}(m)} \sum_{\substack{n \leqslant x \\ p m \mid F(n)}}^{\prime \prime} 1,
$$

où la double apostrophe signifie que : $p^{\prime}\left|F(n) \Rightarrow p^{\prime}\right| p m$ ou $p^{\prime}>X$. La somme intérieure relève du lemme 3.4 puisque

$$
p m \leqslant 2 y m \leqslant y^{1-K / 2}
$$

et

$$
P^{+}(p m) \leqslant 2 y \leqslant X^{1 / \vartheta}
$$

On obtient ainsi

$$
S \gg \frac{x}{(\log y)^{r}} \sum_{m \in \mathcal{M}} \chi(m) \tau(m)^{v} \sum_{\log p \in \log y-\mathcal{L}(m)} \frac{\varrho^{*}(p)}{\varphi^{*}(p)} .
$$

Dans la somme intérieure, $p$ parcourt une réunion d'au plus $\tau(m)\left(\leqslant \mathrm{e}^{2 \gamma^{\prime}(v) L}\right)$ intervalles de longueur logarithmique totale $\lambda(m)$ et dont les bornes ont des logarithmes de l'ordre de $\log y$ (puisque $m \leqslant \sqrt{y}$ ). La somme en $p$ est donc

$$
\gg \frac{1}{\log y} \sum_{p} \frac{\varrho^{*}(p) \log p}{p} \gg \frac{\lambda(m)}{\log y}+O\left(\mathrm{e}^{2 \gamma^{\prime}(v) L-c \sqrt{\log y}}\right),
$$

d'après le lemme 3.1. Le terme d'erreur ci-dessus est négligeable compte tenu de la troisième condition $(* *)$ et l'on obtient

$$
S \gg x(\log y)^{\sigma-1-r} \sum_{m \in \mathcal{M}} \chi(m) \tau(m)^{v} .
$$

Le résultat souhaité découle donc de $(6 \cdot 13)$.

Fin de la démonstration du théorème et du complément.

Nous sommes maintenant en mesure d'établir les minorations de (1.13) et $(1 \cdot 14)$ avec $c_{0}=1-K$. 
Soit $c_{11}$ la constante du lemme 6.2 pour le choix de $\varepsilon$ défini à la proposition 6.3. Lorsque

$$
0 \leqslant \beta \leqslant \gamma^{\prime}(0)-1-2 c_{11} / \sqrt{\log _{2} y},
$$

on peut choisir $v=0$ dans $(6 \cdot 8)$. De plus la condition (6.15) n'est non vide que si $\gamma^{\prime}(0)>1$, et donc $\gamma^{\prime}(0) \geqslant \log 3$. Choisissons alors, dans la proposition 6.3, $\beta_{0}<\log 3-1$. Nous pouvons minorer $H_{F}(x, y, z)$ par $H_{F}(x, y, Z)$ avec

$$
\beta_{1}:=\beta(y, Z)=\max \left(\beta, \beta_{0}\right) .
$$

La formule (6.3) montre que l'on a alors $\sigma\left(\beta_{1}, 0\right)=1$. Puisque $\gamma(0)=0$, la formule (6.8) implique

$$
H_{F}(x, y, Z) \gg x
$$

d'où la minoration du point (a) de notre théorème.

Supposons maintenant que l'on a

$$
\max \left(0, \gamma^{\prime}(0)-1-2 c_{11} / \sqrt{\log _{2} y}\right) \leqslant \beta \leqslant \gamma^{\prime}(1)-1-2 c_{11} / \sqrt{\log _{2} y} .
$$

Considérons d'abord le cas où $F$ est réductible. Alors $\gamma^{\prime}(0) \geqslant \log 3$ et l'on a $\beta \geqslant \beta_{0}$ pour une constante positive convenable. Nous déterminons, dans (6.8), le paramètre $v$ par l'équation

$$
\gamma^{\prime}(v)=\beta+1+\frac{2 c_{11}}{\sqrt{\log _{2} y}} .
$$

On a alors $0 \leqslant v \leqslant 1$ et

$$
v \leqslant u+\frac{c_{13}}{\sqrt{\log _{2} y}} .
$$

La formule $(6 \cdot 3)$ donne encore $\sigma=1$, d'où

$$
\begin{aligned}
& \sum_{\substack{n \leqslant x \\
(n): y, z) \geqslant 1}} \tau\left(n_{L}\right)^{v} \gg x(\log y)^{\gamma(v) .} \\
& \tau(F(n) ; y, z) \geqslant 1 \\
& \log \tau\left(n_{L}\right) \leqslant \gamma^{\prime}(v) L+c_{12} \sqrt{L}
\end{aligned}
$$

On en déduit immédiatement

$$
H_{F}(x, y, z) \gg x(\log y)^{\gamma(v)-v \gamma^{\prime}(v)} \mathrm{e}^{-c_{12} v \sqrt{\log _{2} y}} .
$$

La relation (6.17) implique alors l'estimation inférieure de (1·14), i.e.

$$
H_{F}(x, y, z) \gg x(\log y)^{-\delta(\beta, F)} \mathrm{e}^{-c_{4} \sqrt{\log _{2} y}}
$$

puisque $\delta(\beta, F)=u \gamma^{\prime}(u)-\gamma(u)$. 
Lorsque $F$ est irréductible, le même raisonnement s'applique si $\beta \geqslant \beta_{0}>0$. Dans le cas contraire, nous pouvons encore opérer le même choix de $v$, mais la formule $(6 \cdot 3)$ donne seulement cette fois

$$
\sigma \geqslant 1-2 c_{11} \sqrt{\frac{\log _{3} y}{\log _{2} y}} .
$$

Nous obtenons donc comme annoncé

$$
H_{F}(x, y, z) \gg x(\log y)^{-\delta(\beta, F)} \mathrm{e}^{-c_{4} \sqrt{\log _{2} y \log _{3} y}} .
$$

Il reste à examiner le cas où

$$
\gamma^{\prime}(1)-1-2 c_{11} / \sqrt{\log _{2} y} \leqslant \beta \leqslant B .
$$

On choisit alors, dans $(6 \cdot 8), v=1$. Par $(6 \cdot 3)$, on a

$$
\sigma \geqslant \gamma^{\prime}(1)-\beta-2 c_{11} / \sqrt{\log _{2} y}
$$

En majorant, dans $(6 \cdot 8), \tau\left(n_{L}\right)$ par

$$
(\log y)^{\gamma^{\prime}(1)} \mathrm{e}^{2 c_{12} \sqrt{\log _{2} y}},
$$

il vient

$$
H_{F}(x, y, z) \gg x(\log y)^{\gamma(1)-\beta-1} \mathrm{e}^{-c_{14} \sqrt{\log _{2} y}}
$$

d'où l'estimation requise puisque $\gamma(1)=\widehat{\Omega}(F)$.

Ajouté aux épreuves. Nous avons très récemment démontré, par une méthode complètement différente, l'inégalité

$$
H_{F}\left(x, \frac{1}{2} x, x\right)>x(\log x)^{-\eta} \quad(x \rightarrow \infty)
$$

pour tout $\eta>\log 4-1$ et tout polynôme $F(X)$ irréductible dans $\mathbb{Z}[X]-$ cf. G. Tenenbaum, Sur une question d'Erdős et Schinzel, II, Inventiones math. 99 (1990), 215-24. Cela implique en particulier la validité de (1-17) pour toute constante $c$ satisfaisant à $c<2-\log 4 \approx 0,613705$.

\section{Bibliographie}

[1] H. Delange, Sur des formules de Atle Selberg, Acta Arith. 19 (1971), 105-146.

[2] J.-M. Deshouillers et H. Iwanieč, On the greatest prime factor of $n^{2}+1$, Ann. Inst. Fourier (Grenoble) 32,4 (1982), 1-11.

[3] V. Ennola, A note on a divisor problem, Ann. Univ. Turku, Ser. AI, 118-2 (1968), 3-11.

[4] P. Erdős, The sum $\sum d\{f(n)\}$, J. London Math. Soc. 27 (1952), 7-15. 
[5] P. Erdős, On the greatest prime factor of $\prod f(k), J$. London Math. Soc. 27 (1952), 379-384.

[6] P. Erdős et A. Schinzel, On the greatest prime factor of $\prod_{k=1}^{x} f(k)$, Acta Arith. 55, no. 2, 191-200.

[7] H. Halberstam et H.-E. Richert, Sieve Methods, Academic Press, London, NewYork, San Francisco (1974).

[8] R.R. Hall et G. Tenenbaum, Divisors, Cambridge Tracts $n^{\circ}$ 90, Cambridge University Press (1988).

[9] C. Hooley, On the greatest prime factor of a quadratic polynomial, Acta Math. 117 (1967), 2-16.

[10] C. Hooley, Applications of sieve methods to the theory of numbers, Cambridge Tracts $n^{\circ} 70$, Cambridge University Press (1976).

[11] E. Landau, Einführung in die elementäre und analytische Theorie der algebraischen Zahlen, Teubner, Leipzig (1927); réimpression : Chelsea, New York (1949).

[12] S. Lang, Algebraic Number Theory, Addison-Wesley, Reading, Menlo Park, London, Don Mills (1970).

[13] H. Maier et G. Tenenbaum, On the set of divisors of an integer, Invent. Math. 76 (1984), 121-128.

[14] A. A. Markov, Über die Primteiler der Zahlen von der Form $1+4 x^{2}$, Bull. Acad. Sci. St. Petersburg, 3 (1895), 55-59.

[15] T. Nagell, Introduction to Number Theory, Chelsea, New York (1964)

[16] A. Selberg, Note on a paper by L.G. Sathe, J. Indian Math. Soc. 18 (1954), 83-87.

[17] P. Shiu, A Brun-Titchmarsh theorem for multiplicative functions, J. reine angew. Math. 313 (1980), 161-170.

[18] G. Tenenbaum, Sur la probabilité qu'un entier possède un diviseur dans un intervalle donné, Compositio Math. 51 (1984), 243-263.

[19] D. Wolke, Multiplikative Funktionen auf schnell wachsenden Folgen, J. reine angew. Math. 251 (1971), 54-67.

[20] D. Wolke, Polynomial values with small prime divisors, Acta Arith. 19 (1971), 327333.

Gérald Tenenbaum

Institut Élie Cartan

Université Henri Poincaré-Nancy 1

BP 239

54506 Vandouvre Cedex

France 